\title{
STABILIZATION OF THE WAVE EQUATION BY ON-OFF AND POSITIVE-NEGATIVE FEEDBACKS *
}

\author{
Patrick Martinez ${ }^{1}$ And Judith VAncostenoble ${ }^{1}$
}

\begin{abstract}
Motivated by several works on the stabilization of the oscillator by on-off feedbacks, we study the related problem for the one-dimensional wave equation, damped by an on-off feedback $a(t) u_{t}$. We obtain results that are radically different from those known in the case of the oscillator. We consider periodic functions $a$ : typically $a$ is equal to 1 on $(0, T)$, equal to 0 on $(T, q T)$ and is $q T$-periodic. We study the boundary case and next the locally distributed case, and we give optimal results of stability. In both cases, we prove that there are explicit exceptional values of $T$ for which the energy of some solutions remains constant with time. If $T$ is different from those exceptional values, the energy of all solutions decays exponentially to zero. This number of exceptional values is countable in the boundary case and finite in the distributed case. When the feedback is acting on the boundary, we also study the case of postive-negative feedbacks: $a(t)=a_{0}>0$ on $(0, T)$, and $a(t)=-b_{0}<0$ on $(T, q T)$, and we give the necessary and sufficient condition under which the energy (that is no more nonincreasing with time) goes to zero or goes to infinity. The proofs of these results are based on congruence properties and on a theorem of Weyl in the boundary case, and on new observability inequalities for the undamped wave equation, weakening the usual "optimal time condition" in the locally distributed case. These new inequalities provide also new exact controllability results.
\end{abstract}

Mathematics Subject Classification. 35L05, 35B35, 35B40, $11 \mathrm{~A} 07$.

Received June 25, 2001. Revised November 27, 2001.

\section{Contents}

1. Introduction $\quad 336$

2. Main results $\quad 338$

2.1. Main results for boundary on-off dampings 338

2.2. Main results for boundary positive-negative feedbacks 339

2.3. Main results for locally distributed on-off feedbacks 340

2.4. "On-off" observability inequalities 340

3. Relation to literature 341

3.1. The time-independent case 341

3.2. The time-dependent case $\quad 342$

3.3. The on-off case $\quad 342$

Keywords and phrases: Damped wave equation, asymptotic behavior, on-off feedback, congruences, observability inequalities.

* This work was partially made when the first author was working in the ENS Cachan, Antenne de Bretagne.

1 M.I.P. Université Paul Sabatier Toulouse III, 118 route de Narbonne, 31062 Toulouse Cedex 4, France;

e-mail: martinez@mip.ups-tlse.fr, vancoste@mip.ups-tlse.fr 
4. Comments on the results and optic rays propagation 343

4.1. Comments in the case of boundary feedback 343

4.2. Comments in the case of locally distributed feedback 344

5. Other results and comments on the proofs 344

5.1. Other results in the case of a boundary on-off feedback 344

5.2. Remarks on the proof in the case of a locally distributed feedback 346

6. Proof of Proposition 5.1 (on-off boundary feedback when $q=2$ ) 350

6.1. The useful formula for the energy 350

6.2. Proof of Proposition 5.1 351

7. Proof of Theorem 2.1 (on-off boundary feedback in the general case) 353

7.1. Stability if $\mathrm{T}$ is different of some exceptional values 353

7.2. Non stability if $\mathrm{T}$ is equal to one of those exceptional values 353

8. Proof of Theorem 2.2 (positive-negative boundary feedbacks) 354

8.1. The cyclic case: $\mathbf{1} / \boldsymbol{T} \in \mathbb{Q} \backslash \mathbb{N} \quad 355$

8.2. The equidistributed case: $\mathbf{1} / \boldsymbol{T} \notin \mathbb{Q} \quad 356$

9. Proof of Proposition 5.2 (estimate of the extinction time) 358

10. Proof of Theorem 2.3 when $q=2$ (locally distributed on-off feedback) 361

10.1. The useful tool $\quad 361$

10.2. Proof of Theorem 2.3 when $q=2$ and $\boldsymbol{\omega}=(\mathbf{0}, \mathbf{1}) \quad 362$

10.3. Proof of Theorem 2.3 when $\mathrm{q}=2$ and $\boldsymbol{\omega}=(\mathbf{1} / \mathbf{2}-\boldsymbol{\lambda}, \mathbf{1} / \mathbf{2}+\boldsymbol{\lambda})$ in the case $\mathbf{1} / \boldsymbol{T} \notin \mathbf{2} \mathbb{N} \quad 363$

10.4. Proof of Theorem 2.3 when $\mathrm{q}=2$ and $\boldsymbol{\omega}=(\mathbf{1} / \mathbf{2}-\boldsymbol{\lambda}, \mathbf{1} / \mathbf{2}+\boldsymbol{\lambda})$ in the case $\mathbf{1} / \boldsymbol{T} \in \mathbf{2} \mathbb{N}$ and

$$
2 \lambda<T \quad 365
$$

10.5. Proof of Theorem 2.3 when $q=2$ and $\boldsymbol{\omega}=(\mathbf{1} / \mathbf{2}-\boldsymbol{\lambda}, \mathbf{1} / \mathbf{2}+\boldsymbol{\lambda})$ and $\mathbf{1} / \boldsymbol{T} \in \mathbf{2} \mathbb{N}$ and $\mathbf{2} \boldsymbol{\lambda}>\boldsymbol{T} \quad 366$

11. Proof of Theorem 5.2 (observability inequalities) 366

11.1. Proof of Lemma 11.1 368

11.2. $\quad$ Proof of Lemma 11.2 371

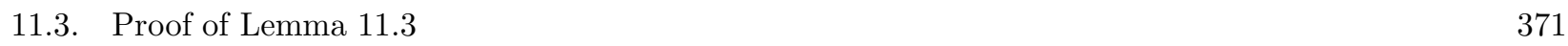

11.4. Proof of Lemma 11.4 373

11.5. Proof of Lemma 11.5

12. Tools for the proof of Theorem 2.3 and Theorem $2.4 \quad 373$

13. Proof of Proposition 10.1 (link between stabilization and observability) 374

13.1. Equation (10.3) implies (10.4) 374

13.2. $\quad$ Equation (10.4) implies (10.3) 375

$\begin{array}{ll}\text { References } & 376\end{array}$

\section{InTRODUCTION}

Motivated by several works on ordinary differential equations $[1,11,12,31,34,35]$, we consider first the wave equation in one space dimension, damped by a boundary on-off feedback $a(t) u_{t}$, where $a: \mathbb{R}_{+} \longrightarrow \mathbb{R}_{+}$is a bounded nonnegative function that can sometimes be equal to zero:

$$
\begin{cases}u_{t t}-u_{x x}=0, & x \in(0,1), t \geq 0 \\ u(0, t)=0, & t \geq 0 \\ u_{x}(1, t)=-a(t) u_{t}(1, t), & t \geq 0 \\ \left(u(x, 0), u_{t}(x, 0)\right)=\left(u^{0}(x), u^{1}(x)\right), & x \in(0,1)\end{cases}
$$

where $\left(u^{0}, u^{1}\right)$ is given in $V \times L^{2}(0,1)$ (with $\left.V=\left\{v \in H^{1}(0,1) \mid v(0)=0\right\}\right)$. 
Next we consider the wave equation in one space dimension, damped by a locally distributed on-off feedback $a(t) \chi_{\omega}(x) u_{t}$, where $\omega \subset(0,1)$ :

$$
\begin{cases}u_{t t}-u_{x x}=-a(t) \chi_{\omega}(x) u_{t}, & x \in(0,1), t \geq 0 \\ u(0, t)=u(1, t)=0, & t \geq 0 \\ \left(u(x, 0), u_{t}(x, 0)\right)=\left(u^{0}(x), u^{1}(x)\right), & x \in(0,1)\end{cases}
$$

where $\left(u^{0}, u^{1}\right)$ is given in $H_{0}^{1}(0,1) \times L^{2}(0,1)$.

In both cases we define the energy of $u$ by

$$
\forall t \geq 0, \quad E_{u}(t)=\frac{1}{2} \int_{0}^{1}\left(u_{x}^{2}(x, t)+u_{t}^{2}(x, t)\right) \mathrm{d} x .
$$

We are interested in the asymptotic behavior of the energy. We consider the typical case where

$$
a(t)=a_{0}>0 \text { for } t \in[0, T), a(t)=0 \text { for } t \in[T, q T) \text { and } a \text { is } q T \text {-periodic }
$$

for some integer $q \geq 2$. In both cases, we prove that there is a set of explicit exceptional values for $T$ for which the energy of some solutions remains constant with time. If $T$ is not one of those exceptional values, the energy decays exponentially to zero (and the more $T$ is close to such an exceptional value, the slowlier it decays). This set is countable in the boundary case, given by

$$
\frac{1}{T} \in \bigcup_{p=1}^{q-1} \frac{q}{2 p} \mathbb{N}
$$

and only finite in the locally distributed case: if $\omega=((1 / 2)-\lambda,(1 / 2)+\lambda) \subset(0,1)$, it is given by

$$
\frac{1}{T} \in \bigcup_{p=1}^{q-1} \frac{q}{p} \mathbb{N} \text { and }(q-1) T>2 \lambda .
$$

We also characterize (in term of support) the initial conditions that create solutions whose energy does not decay to zero.

Motivated by a question of Zuazua and [7], we consider also positive-negative feedbacks for (1.1): $a$ is $q T$ periodic and

$$
a(t)=a_{0}>0 \text { for } t \in[0, T), a(t)=-b_{0}<0 \text { for } t \in[T, q T) .
$$

Note that in this case the energy is only nonincreasing during the time intervals $[m q T, m q T+T)$, and nondecreasing during the other time intervals. If $T$ is exceptional, then the energy of some solutions increases to infinity. If $T$ is not exceptional, we give the necessary and sufficient condition that tells that, roughly speaking, the energy of all solutions goes exponentially to zero, or the energy of some solutions go exponentially to infinity: for example if $1 / T \notin \mathbb{Q}$, then denoting

$$
K_{0}:=\left(\frac{a_{0}-1}{a_{0}+1}\right)^{1 / q}\left(\frac{b_{0}+1}{b_{0}-1}\right)^{(q-1) / q}
$$

stability holds if and only if $K_{0}<1$. In particular, note that stability always holds if $a_{0}=1$ (for all $b_{0} \neq 1$ ).

For the boundary case, our proofs are based on congruence properties and a theorem of Weyl. For the distributed case, they are based on new observability inequalities: "on-off" observability inequalities, that tells 
that, roughly speaking, if $T_{0}$ is the minimal time that one need to observe the solution, then observing it "half of the time" can be sufficient (in fact only some parts of the time interval $\left(0, T_{0}\right)$ are useful).

Our results are radically different from those of the wave equation damped by linear time-dependent feedbacks $a(t) u^{\prime}$ when $a$ is always positive (possibly decaying to zero), and even radically different from those of the ordinary differential equations damped by on-off feedbacks.

More precisely, our results are the following:

\section{MAin RESUltS}

\subsection{Main results for boundary on-off dampings}

First consider the problem (1.1) and the following function $a$ :

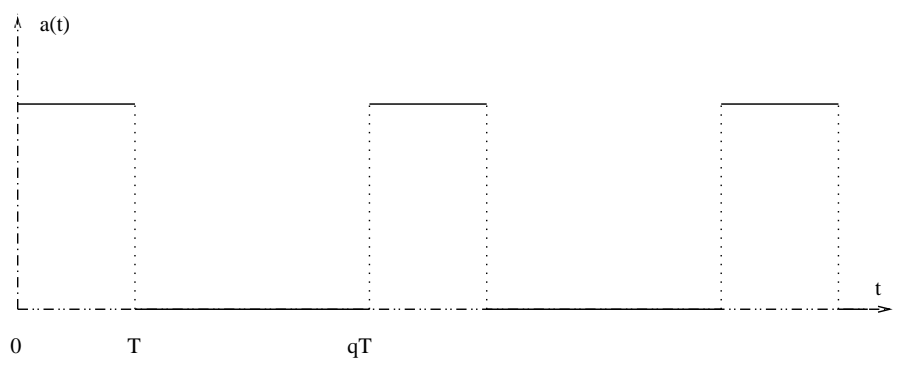

FIGURE 1.

Such on-off feedback laws have been widely studied in the case of ordinary differential equations, but seem to have never been studied for partial differential equations. We prove the following:

Theorem 2.1. Assume (1.3). For all $\left(u^{0}, u^{1}\right) \in V \times L^{2}(0,1)$, there exists a unique $u$ solution of (1.1). Moreover,

(i) if

$$
\frac{1}{T} \in \bigcup_{p=1}^{q-1} \frac{q}{2 p} \mathbb{N}
$$

there exists some $\left(u^{0}, u^{1}\right) \in V \times L^{2}(0,1)$ such that $E_{u}(t)$ remains constant with time: $E_{u}(t)=E_{u}(0)>0$ for all $t \geq 0$;

(ii) if

$$
\frac{1}{T} \notin \bigcup_{p=1}^{q-1} \frac{q}{2 p} \mathbb{N}
$$

then for all $\left(u^{0}, u^{1}\right) \in V \times L^{2}(0,1)$, the energy $E_{u}(t)$ of the solutions of (1.1) decays uniformly exponentially to 0 (or achieves zero in finite time in the particular case $a_{0}=1$ ).

\section{Remarks.}

1. The non stability result in the exceptional case (2.1) is radically different from what happens for ordinary differential equations, or even for partial differential equations like (1.1) when the function $a$ decreases to zero remaining always positive (see [25]). 
2. We also characterize (in term of support) the initial conditions that create solutions whose energy does not decay to zero. Moreover, in the non-exceptional case (2.2), we provide estimates on the "extinction time" (where the energy is equal to zero), and we give optimal estimates when $q=2$ and $q=3$.

3. We also give explicit and optimal estimate of the exponent of the exponential decay of the energy or of the extinction time in the particular case $a_{0}=1$. See Section 5 .

\subsection{Main results for boundary positive-negative feedbacks}

Now we consider the more general case of positive-negative feedbacks: the function $a$ is now $2 T$-periodic to simplify:

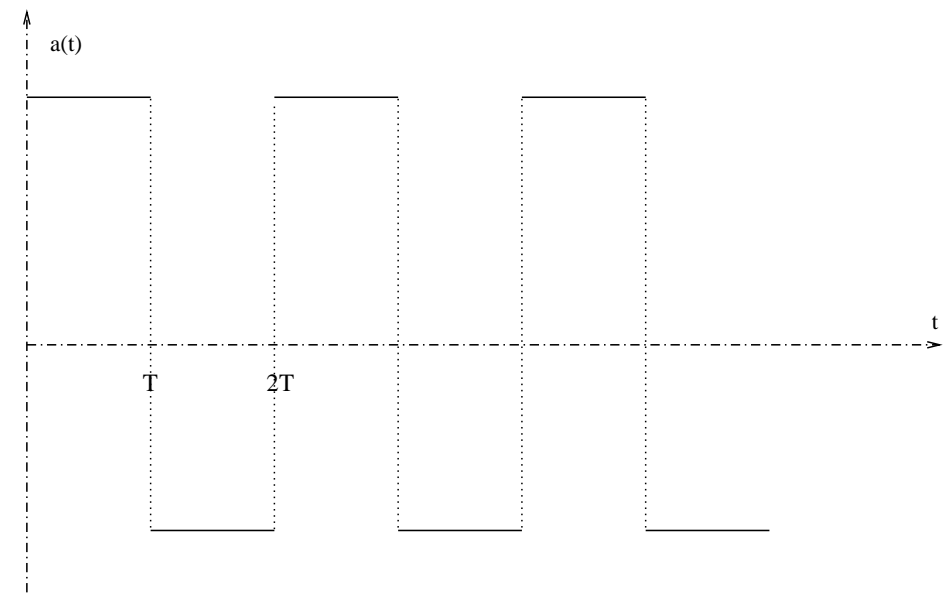

FIGURE 2.

In this case, note that the energy is only nonincreasing on the time intervals $[2 m T, 2 m T+T)$ and nondecreasing the other time intervals. There are some works when the feedback is of the type $b(x) u^{\prime}$, where the function $b$ depends on $x$ (and not on $t$ ) and is of indefinite sign, but "more positive than negative" (see, e.g., Freitas and Zuazua [7], Benaddi and Rao [5]) But to our knowledge, such time dependent positive-negative feedbacks laws have never being studied. We prove the following:

Theorem 2.2. Assume (1.4) with $q=2$. Assume that $b_{0} \neq 1$. Then for all $\left(u^{0}, u^{1}\right) \in V \times L^{2}(0,1)$, there exists a unique $u$ solution of (1.1). Moreover,

(i) if $T$ satisfies $(2.1)$, that is simply $1 / T \in \mathbb{N}$ in this case $(q=2)$, then there exists some $\left(u^{0}, u^{1}\right)$ $\in V \times L^{2}(0,1)$ such that $E_{u}(t)$ goes exponentially to infinity as $t \rightarrow \infty$;

(ii) if $1 / T=p^{\prime} / q^{\prime}$ where $p^{\prime}$ and $q^{\prime}$ are relatively primes, denote

$$
\left\{\begin{array}{l}
K_{T}:=\left(\frac{a_{0}-1}{a_{0}+1}\right)^{1 / 2}\left(\frac{b_{0}+1}{b_{0}-1}\right)^{1 / 2} \text { if } q^{\prime} \text { is even, } \\
K_{T}:=\left(\frac{a_{0}-1}{a_{0}+1}\right)^{\left(q^{\prime}-1\right) / 2 q^{\prime}}\left(\frac{b_{0}+1}{b_{0}-1}\right)^{1-\left(q^{\prime}-1\right) / 2 q^{\prime}} \text { if } q^{\prime} \text { is odd }
\end{array}\right.
$$

then if $K_{T}<1$, the energy of all solutions goes exponentially to zero (as $K_{T}^{t}$ ), an if $K_{T}>1$, the energy of some solutions goes exponentially to infinity $\left(\right.$ as $\left.K_{T}^{t}\right)$;

(iii) if $1 / T \notin \mathbb{Q}$, denote

$$
K_{0}:=\left(\frac{a_{0}-1}{a_{0}+1}\right)^{1 / 2}\left(\frac{b_{0}+1}{b_{0}-1}\right)^{1 / 2}
$$


then if $K_{0}<1$, the energy of all solutions goes exponentially to zero (as $K_{0}^{t}$ ), an if $K_{0}>1$, the energy of some solutions goes exponentially to infinity (as $K_{0}^{t}$ ).

\section{Remarks.}

1. It is interesting to note that if $a_{0}=1$, then for all values of $b_{0}$ (except $b_{0}=1$ for which we have no solution), we obtain exponential decay.

2. Part (iii) relies on a theorem of Weyl: if $\theta \notin \mathbb{Q}$, then the sequence $(\{n \theta\})_{n}$ is not only dense but also equidistributed in [0,1). (As usual, $\{x\}$ denotes the fractional part of $x$.) Consequently, the critical value $K_{0}$ that appears in (iii) does not depend on $T$.

3. Theorem 2.2 could easily be extended to the general case $q \geq 2$; the critical value in part (iii) would become

$$
\tilde{K}_{0}:=\left(\frac{a_{0}-1}{a_{0}+1}\right)^{1 / q}\left(\frac{b_{0}+1}{b_{0}-1}\right)^{1-1 / q} .
$$

\subsection{Main results for locally distributed on-off feedbacks}

Now we consider the problem $(1.2)$, where $\omega$ is the open nonempty subset $((1 / 2)-\lambda,(1 / 2)+\lambda)$ of $(0,1)$, a is the time periodic function (1.3) and $\left(u^{0}, u^{1}\right)$ is given in $H_{0}^{1} \times L^{2}(0,1)$. We prove the following:

Theorem 2.3. Assume (1.3) and assume that $0<\lambda \leq 1 / 2$.

(i) If

$$
\frac{1}{T} \in \bigcup_{p=1}^{q-1} \frac{q}{p} \mathbb{N} \text { and }(q-1) T>2 \lambda
$$

then there exists initial conditions $\left(u^{0}, u^{1}\right) \in H_{0}^{1} \times L^{2}(0,1)$ such that the energy of the solutions of (1.2) remains constant with time: $E_{u}(t)=E_{u}(0)>0$ for all $t \geq 0$.

(ii) If

$$
\left(\frac{1}{T} \in \bigcup_{p=1}^{q-1} \frac{q}{p} \mathbb{N} \quad \text { and } \quad(q-1) T<2 \lambda\right), \quad \text { or } \quad\left(\frac{1}{T} \notin \bigcup_{p=1}^{q-1} \frac{q}{p} \mathbb{N}\right)
$$

then the energy of the solutions of (1.2) decays uniformly exponentially to 0.

Once again, this is radically different from what happens for ordinary differential equations, or even for the damped wave equation when the function $a$ decreases to zero remaining always positive. In the case (2.5), we prove the uniform decay of the energy thanks to new observability inequalities:

\section{4. "On-off" observability inequalities}

Considering the undamped problem

$$
\begin{cases}\phi_{t t}-\phi_{x x}=0, & x \in 0,1), t \geq 0 \\ \phi(0, t)=\phi(1, t)=0, & t \geq 0 \\ \left(\phi(x, 0), \phi_{t}(x, 0)\right)=\left(\phi^{0}(x), \phi^{1}(x)\right), & x \in(0,1)\end{cases}
$$

it is well known that if $0<a<b<1$ and $T^{*}>2 \max (a, 1-b)$, then the solutions of (2.6) satisfy the following observability inequality

$$
E_{\phi}(0) \leq C \int_{0}^{T^{*}} \int_{a}^{b} \phi_{t}^{2}(x, t) \mathrm{d} x \mathrm{~d} t
$$


for some positive constant $C=C\left(T^{*}\right)$ (see Haraux [9] and Zuazua [37]). This is optimal in the sense that you cannot have this inequality with some $T^{*}<2 \max (a, 1-b)$. In our case, for example, if $\lambda>1 / 8$, then we can apply it with $T^{*}=3 / 4$ and we obtain

$$
E_{\phi}(0) \leq C \int_{0}^{3 / 4} \int_{(1 / 2)-\lambda}^{(1 / 2)+\lambda} \phi_{t}^{2}(x, t) \mathrm{d} x \mathrm{~d} t
$$

We improve this inequality showing that

$$
E_{\phi}(0) \leq C \int_{0}^{1 / 4} \int_{(1 / 2)-\lambda}^{(1 / 2)+\lambda} \phi_{t}^{2}(x, t) \mathrm{d} x \mathrm{~d} t+C \int_{1 / 2}^{3 / 4} \int_{(1 / 2)-\lambda}^{(1 / 2)+\lambda} \phi_{t}^{2}(x, t) \mathrm{d} x \mathrm{~d} t .
$$

This is coherent with the fact that each optic ray touches the damping region during the time intervals $(0,1 / 4)$ or $(1 / 2,3 / 4)$.

More generally, we prove the following:

Theorem 2.4. Assume $1 / T \in \bigcup_{p=1}^{q-1} \frac{q}{p} \mathbb{N}$ and $(q-1) T<2 \lambda$. Then there exists $C>0$ such that, for all $\phi$ solution of (2.6),

$$
E_{\phi}(0) \leq C \int_{0}^{q-1} a(t) \int_{\omega} \phi_{t}^{2}(x, t) \mathrm{d} x \mathrm{~d} t
$$

Note that this obviously also gives new exact controllability results, applying the method H.U.M. of J.-L. Lions [22]: given $\left(u^{0}, u^{1}\right) \in H_{0}^{1}(\Omega) \times L^{2}(\Omega)$, there exists a control $h \in L^{2}\left((0,1 / 4) \times(1 / 2,3 / 4), L^{2}(\omega)\right)$ such that the solution of the problem

$$
\begin{cases}u_{t t}-u_{x x}=\chi_{\omega}(x) h(t, x), & x \in(0,1), t \geq 0 \\ u(0, t)=u(1, t)=0, & t \geq 0 \\ \left(u(x, 0), u_{t}(x, 0)\right)=\left(u^{0}(x), u^{1}(x)\right), & x \in(0,1)\end{cases}
$$

satisfies $u(3 / 4)=0=u_{t}(3 / 4)$. This implies that the control $h$ that drives the system from the state $\left(u^{0}, u^{1}\right)$ to the rest has only to act on the time intervals $(0,1 / 4)$ and $(1 / 2,3 / 4)$. Our observability inequalities or exact controllability results are coherent with the general results related on the rays propagation for systems with time independent coefficients (see [2,3]). Moreover we provide a direct proof of these inequalities, and we would like to emphasize that our method can also provide results for semilinear wave equations (see [26]).

\section{Relation to literature}

\subsection{The time-independent case}

We are interested in the asymptotic behavior of the energy of the solutions of (1.1). First we recall that if the function $a$ is constant:

$$
a(t)=a_{0}>0 \text { for all } t \in \mathbb{R}_{+},
$$

then for all $\left(u^{0}, u^{1}\right)$ given in $V \times L^{2}(0,1)$, there exists a unique solution $u$ of $(1.1)$ and its energy $E_{u}(t)$ decays exponentially to 0 as $t$ goes to infinity. More precisely, it is easy to prove that:

- if $a_{0}=1$, then $E_{u}(t)$ achieves 0 in finite time in time $t=2: E_{u}(2)=0$;

- if $a_{0} \neq 1$ then $E_{u}(t) \rightarrow 0$ exponentially as $t \rightarrow \infty$ with an explicit decay rate that depends on $a_{0}$ :

$$
\forall t \geq 0, E_{u}(t) \leq E_{u}(0) \mathrm{e}^{-\omega(t / 2-1)} \text { with } \omega=2 \ln \left|\frac{a_{0}+1}{a_{0}-1}\right|>0 .
$$


On the other hand, with the same feedback $a(t)=a_{0}>0$ for all $t \in \mathbb{R}_{+}$, exponential decay of the solutions of (1.2) follows easily from the multiplier method.

Of course, in both cases, the exponential decay of the energy for this problem is also an easy consequence of the general "optic rays condition" of Bardos et al. [2]: it is clear that each optic ray touches the damping region (the point 1) in time at most 2 in the case of boundary damping, and crosses the region $\omega$ in time at most 2 in the case of locally distributed damping.

Remark. The proof of the extinction in finite time follows directly from D'Alembert Formula (see the beginning of the proofs of Th. 2.1, (ii) or Prop. 5.1). Note that extinction in finite time was proved in [16] (see Th. 0.5, p. 6): for the wave equation with a boundary feedback acting at both extremities $(x=0$ and $x=1)$, Komornik obtained the extinction in time $t=1$. In our case, since the feedback only acts at one extremity $(x=1)$, we have the same phenomenon but we need twice more time before the extinction.

Note that this phenomenon is related to the radiation boundary conditions (see [4]), which correspond to the fact that there is no reflection from the boundary into the domain. Indeed, in the case of the wave equation, the boundary condition $u_{x}(1, t)+u_{t}(1, t)=0$ is a radiation boundary condition.

\subsection{The time-dependent case}

Now we consider the asymptotic behavior of the energy under time-dependent feedback laws. This problem has been largely studied when the damping term is "not too small" (in order to prevent underdamping) or "not too large" (to prevent overdamping), that means when there exists some positive and nonincreasing continuous function $\sigma: \mathbb{R}_{+} \longrightarrow \mathbb{R}_{+}$that satisfies

$$
\begin{gathered}
\forall t \geq 0, \quad \sigma(t) \leq a(t) \leq \frac{1}{\sigma(t)} \\
\int_{0}^{+\infty} \sigma(\tau) \mathrm{d} \tau=\infty
\end{gathered}
$$

In this case, for both problem (1.1) and problem (1.2), the energy decays with an explicit decay rate: there exists $\omega>0$ such that

$$
\forall t \geq 0, \quad E_{u}(t) \leq E_{u}(0) \mathrm{e}^{1-\omega \int_{0}^{t} \sigma(\tau) \mathrm{d} \tau}
$$

On the other hand, if $\int_{0}^{+\infty} a(\tau) \mathrm{d} \tau<\infty$, there are some solutions whose energy does not decay to 0 . (For more general results about stabilization properties using nonlinear time-dependent dampings $g\left(t, u_{t}\right)$, see, e.g. $[25,27,32]$ and the references therein.)

\subsection{The on-off case}

Here we are interested in the asymptotic behavior of the energy under on-off dampings: we assume that the function a may vanish on non-zero measure sets, and we want to study the asymptotic behavior of the energy. To our knowledge, such works have not yet been done in the case of partial differential equations. Bardos et al. [2] (p. 1029) noted just that invisible solutions may appear in the case of time-dependent coefficients, and their existence has to be studied on each case. Note also that in our case the family of invisible solutions is not at all finite dimensional.

However this has been widely studied in the case of ordinary differential equations (see $[1,11,12,31,34,35]$ ). The typical problem is the oscillator damped by an on-off damping

$$
u^{\prime \prime}+a(t) u^{\prime}+u=0
$$


the energy decays to zero if the damping is "sufficiently active": in the case where the function $a$ is equal to 1 on a sequence of disjoint intervals $I_{n}$ and nonnegative elsewhere, then the energy decays to 0 if

$$
\sum_{n}\left|I_{n}\right|^{3}=\infty,
$$

and 3 is the best possible exponent (see [31]). In particular, this implies that under (3.5), the localization of the damping time intervals $I_{n}$ is not important; Pucci and Serrin noted that "the exact switching times can be of great importance" (p. 831 in [31]) in some situations where (3.5) is not satisfied but 0 is still a global attractor of the problem if the switching times are correctly set. More general cases are studied, in particular the case where the function $a$ is equal to some positive constant $m_{n}$ on $I_{n}$, and then 0 is a global attractor of the problem if some condition like (3.5) is satisfied (the condition lies on the divergence of some series whose general term contains $\left|I_{n}\right|$ and $\min \left(m_{n}, \frac{1}{m_{n}}\right)$ in order to prevent underdamping and overdamping).

The case of partial differential equations damped by on-off dampings (applied at the boundary or locally distributed in the domain) is radically different from the case of ordinary differential equations: even in the simplest case where the function $a$ is equal to 1 on $[0, T)$ and to 0 on $[T, 2 T)$, and is $2 T$-periodic, there are values of $T$ for which the energy does not decay to zero, although the conditions $\int_{0}^{+\infty} a(\tau) \mathrm{d} \tau=\infty$ and $\sum_{n}\left|I_{n}\right|^{3}=\infty$ (where $I_{n}:=(2 n T,(2 n+1) T)$ are clearly satisfied.

\section{Comments ON The RESUlts AND OptiC RAYS PROPAGATION}

\subsection{Comments in the case of boundary feedback}

Our proof is based on D'Alembert formula and on congruence properties. In fact this congruence properties are equivalent to study the optic rays propagation; we prove that if $T$ is not one of those exceptional values, each ray touches the boundary point $x=1$ (where the dissipative condition is applied) in time at most $2 N_{T}+2$, where $N_{T}$ depends on $T$ and at an instant time $t$ where the damping is effective. In Figure 3 we present an example where the value of $T$ is exceptional $(T=1 / 5)$. We can see that there exists a ray that touches the boundary only at times when the feedback is non active (or of the wrong sign in the case of positive-negative feedbacks):

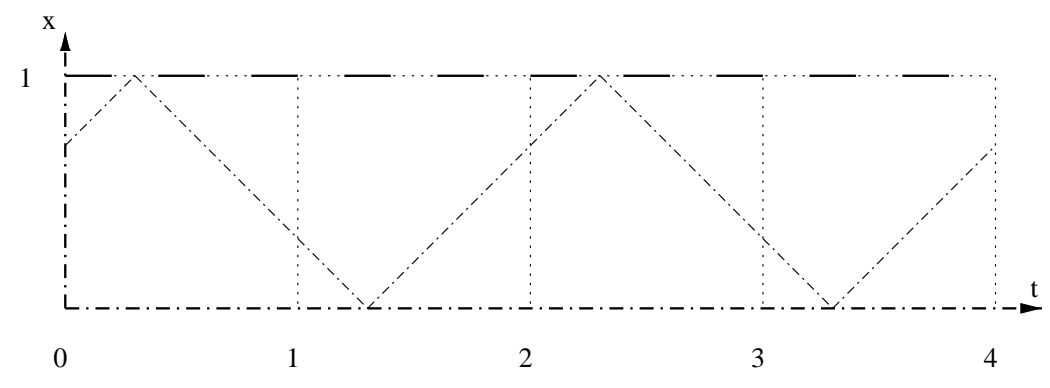

FiguRE 3.

In Figure 4, we present an example where the value of $T$ is not exceptional $(T=2 / 5)$. In this case, we can see that each ray will touch the damping region at a time when the feedback is active.

This property is quite natural with respect to the general optic rays condition for time independent feedbacks [2]. However, we do not know if this theory can be adapted in this case of a time dependent feedback $a(t) u_{t}(1, t)$ (and moreover with a function $a$ that is not continuous), and even if it is true, it is not easy to determine explicitly the exceptional values of $T$. 


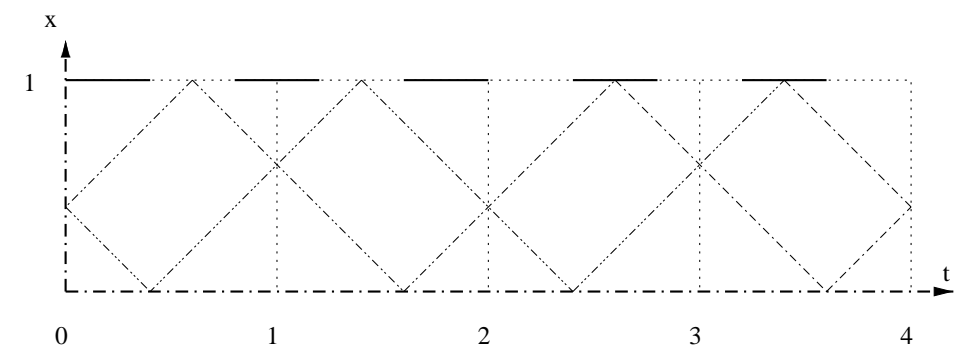

FiguRE 4.

\subsection{Comments in the case of locally distributed feedback}

We are interested in the asymptotic behavior of the energy $E_{u}$ of the solutions of (1.2). First we recall that if the function $a$ is constant on $\mathbb{R}_{+}$, then $E_{u}$ decays exponentially to 0 , for all nonempty open subset $\omega$ wherever its location in $(0,1)$ (see, e.g. [8]). A geometrical explanation of this is that each optic ray touches the damping region $\omega$ in time at most 2 (see [3]). (Note that in the case of a symmetrical open subset $\omega:=(1 / 2-\lambda, 1 / 2+\lambda$ ) with $0<\lambda \leq 1 / 2$, each optic ray touches the damping region in time at most 1 ).

Here we consider the case where $a$ is the periodic function defined by (1.3) and where $\omega:=(1 / 2-\lambda, 1 / 2+\lambda)$ with $0<\lambda \leq 1 / 2(\lambda=1 / 2$ means that the feedback is uniformly distributed in $(0,1))$. There is no problem for the existence and the regularity of the solution, solving successively on the time intervals $(0, T),(T, 2 T), \ldots$

First consider the case of a uniformly distributed damping, i.e. we assume $\lambda=1 / 2$. In this case, note that it is clear that each optic ray crosses the damping region during a period when the damping is effective. In this case, we prove positive results of exponential stabilization.

Next consider the more interesting case of a locally distributed damping: $0<\lambda<1 / 2$. Note carefully that there are some values of $T$ and some values of $\lambda$ for which some rays cross the damping region when the feedback is non active. For example, take $T=1 / 2, \lambda<T / 2=1 / 4$, and consider the optic rays that leaves the point $x=T / 2=1 / 4$ and that goes to the left (towards the point $x=0$ ) at time $t=0$ : this ray describes the segment $[1 / 4,3 / 4]$ (that contains the dissipative region) in direct sens or in the other sens during the time intervals $[T, 2 T],[3 T, 4 T], \ldots$, thus during periods when $a(t)=0$. The same situation occurs if $1 / T \in 2 \mathbb{N}$ with $2 \lambda<T$. We obtain negative results of exponential stabilization in all these cases, and positive results in the other cases, which is coherent with the optic rays condition known for time independent feedbacks [2].

Note that the situation is much more complex than the case of boundary damping: even when $T$ takes some "exceptional" values, we can still have exponential decay of the energy of the solutions, provided that the damping region is large enough. Note also that when the damping region is "large enough" (in particular when $\omega=(0,1)$, then we find a result analogous to the one related to ordinary differential equation, since we obtain stabilization for all $T>0$.

At last, note that the previous positive results of uniform exponential stabilization are still true if we just assume that $a \in L^{\infty}\left(\mathbb{R}_{+} ; \mathbb{R}_{+}\right)$is $q T$-periodic such that $a(t) \geq a_{0}>0$ for $t \in[0, T)$. On the same way, the negative results of uniform exponential stabilization are still true if we just assume that $a \in L^{\infty}\left(\mathbb{R}_{+} ; \mathbb{R}_{+}\right)$is $q T$-periodic such that $a(t)=0$ for $t \in[T, q T)$.

\section{Other RESUlts AND COMMENTS ON THE PROOFS}

\subsection{Other results in the case of a boundary on-off feedback}

\subsubsection{Optimal estimates of the extinction time}

We complete Theorem 2.1 giving optimal estimates of the "extinction time" $\tau_{T}=2 N_{T}+2$, where the energy achieves zero in the particular case $a_{0}=1$. Note that this also give optimal estimate of the exponential energy 
decay in the general case since it is given by

$$
\forall t \geq 0, E_{u}(t) \leq E_{u}(0) \mathrm{e}^{-\omega\left(t /\left(2 N_{T}+2\right)-1\right)} .
$$

We denote by $d(x, \mathbb{N})$ the distance between $x$ and $\mathbb{N}$. We assume that $T<2$ since if $T \geq 2$, then we already know that $E_{u}(t)=0$ for all $t \geq 2$.

First consider the typical case $q=2$. We recall from Theorem 2.1 that if $1 / T \in \mathbb{N}$, the energy of some solutions does not decay to zero. In the other case, we give optimal estimates on the "extinction time":

Proposition 5.1. Assume (1.3) with $q=2$. Then if $1 / T \notin \mathbb{N}$, then for all $\left(u^{0}, u^{1}\right) \in V \times L^{2}(0,1), E_{u}(t)$ achives zero in finite time. More precisely,

$$
\forall t \geq 2 N_{T}+2, E_{u}(t)=0,
$$

where $N_{T}$ is the smallest integer such that

$$
2 N_{T} d\left(\frac{1}{T}, \mathbb{N}\right) \geq 1
$$

Moreover, $N_{T}$ is optimal in the following sense: there exists some $\left(u^{0}, u^{1}\right) \in V \times L^{2}(0,1)$ such that $E_{u}\left(2 N_{T}\right)$ $=E_{u}(0)>0$.

Next we look to the general case. We also give optimal estimates of the "extinction time" when $q=3$ and we give general estimates of the "extinction time" in the general case:

Proposition 5.2. Assume (1.3) and assume (2.2).

(i) Assume that $q=3$. If

$$
d\left(\frac{2}{3 T}, \mathbb{N}\right) \leq \frac{1}{3}
$$

let $N_{T}$ be the smallest integer such that

and if

$$
3 d\left(\frac{2}{3 T}, \mathbb{N}\right) N_{T} \geq 2
$$

$$
\frac{1}{3}<d\left(\frac{2}{3 T}, \mathbb{N}\right)<\frac{1}{2}
$$

let $N_{T}^{\prime}$ be the smallest integer such that

$$
\left(1-2 d\left(\frac{2}{3 T}, \mathbb{N}\right)\right) N_{T}^{\prime} \geq \frac{2}{3}-d\left(\frac{2}{3 T}, \mathbb{N}\right)
$$

and $N_{T}:=2 N_{T}^{\prime}-1$; then $E_{u}(t)=0$ for all $t \geq 2 N_{T}+2$. Moreover, $N_{T}$ is optimal in the following sense: there exists some initial conditions such that $E_{u}\left(2 N_{T}\right)=E_{u}(0)>0$.

(ii) In the general case, we have the following estimate: $E_{u}(t)=0$ for all $t \geq 2 m N+2$, where $m$ and $N$ are integers chosen such that

$$
0<\left\{m\left\{\frac{2}{q T}\right\}\right\} \leq \frac{1}{q} \text { and } N\left\{m\left\{\frac{2}{q T}\right\}\right\} \geq \frac{q-1}{q}
$$

(where $\{x\}$ denotes the fractional part of $x$ ). 
Remark. Such $m$ always exists under (2.2). It would be interesting to find in a general way the best time $\tau_{T}$ for which $E_{u}(t)=0$ if $t \geq \tau_{T}$, like we did for $q=2$ and $q=3$.

\subsubsection{Extension to nonlinear feedbacks}

Combining the methods used in [36] with the method used for the proof of Proposition 5.1, we can also study the wave equation damped by a boundary nonlinear on-off feedback:

$$
\begin{cases}u_{t t}-u_{x x}=0, & x \in(0,1), t \geq 0 \\ u(0, t)=0, & t \geq 0 \\ u_{x}(1, t)=-a(t) g\left(u_{t}(1, t)\right), & t \geq 0 \\ \left(u(x, 0), u_{t}(x, 0)\right)=\left(u^{0}(x), u^{1}(x)\right), & x \in(0,1)\end{cases}
$$

where $a$ is defined by (1.3) and where $g$ is increasing. Once again, when $T$ is exceptional, we have not stability, and when $T$ is not exceptional, we easily prove that there is still uniform stabilization, with explicit decay rate that depends on $g$.

\subsection{Remarks on the proof in the case of a locally distributed feedback}

\subsubsection{Link between on-off stabilization and on-off observability}

To prove Theorem 2.3 in the typical case $q=2$, we first reduce the problem to the proof of an observability inequality for the wave equation without damping (see Prop. 10.1): there is uniform exponential stabilization of solutions of (1.2) if and only if the solutions of (2.6) satisfy, for some $T^{*}>0$,

$$
E_{\phi}(0) \leq C \int_{0}^{T^{*}} a(t) \int_{\omega} \phi_{t}^{2}(x, t) \mathrm{d} x \mathrm{~d} t .
$$

Then to prove this inequality, we will have to distinguish the following different cases:

- $\lambda=1 / 2$ and $T>0$. It is the case of a uniformly distributed damping. In this case, we can easily prove the observability inequality using spectral decomposition of the solutions of the wave equation;

- $\lambda<1 / 2$ and $1 / T \notin 2 \mathbb{N}$. In this case, we use the fact that $T$ is not an exceptional value as we did in the case of boundary feedback, to obtain similar congruence properties. And combining these properties with the spectral decomposition made in the previous case, we deduce the observability inequality;

- $\lambda<1 / 2,1 / T \in 2 \mathbb{N}$ and $2 \lambda<T$. In this case, we construct solutions that never "see the damping" such that the observability inequality does not hold;

- $\lambda<1 / 2,1 / T \in 2 \mathbb{N}$ and $2 \lambda>T$. To treat this case, we need new observability inequalities (see Prop. 5.2) presented in the following section.

\subsubsection{On-off observability inequalities}

Consider the problem (2.6). The following result is well known:

Theorem 5.1 (Haraux [9]). Let $(a, b) \subset(0,1)$ be a given interval. Set

$$
T_{0}^{*}=2 \max (a, 1-b) .
$$

Then for all $T^{*}>T_{0}^{*}$, there exists $C>0$ such that, for all $\phi$ solution of (2.6),

$$
E_{\phi}(0) \leq C \int_{0}^{T^{*}} \int_{a}^{b} \phi_{t}^{2}(x, t) \mathrm{d} x \mathrm{~d} t .
$$


This is optimal in the sense that you cannot have this inequality with some $T^{*}<2 \max (a, 1-b)$. In our case, the condition " $T^{*}>2 \max (a, 1-b)$ " becomes " $T^{*}+2 \lambda>1$ ". Consequently, if we assume $2 \lambda>1-T$, we can directly deduce uniform exponential stabilization from Theorem 5.1.

For example, consider the case $q=2$ and $T=1 / 2$. Note that in this case, the condition " $2 \lambda>T$ " of Theorem 2.3 is exactly the same as the previous condition " $2 \lambda>1-T$ " i.e. it is " $\lambda>1 / 4$ ". So we can apply (5.8) with $T^{*}=T=1 / 2$ and we obtain

$$
E_{\phi}(0) \leq C \int_{0}^{1 / 2} \int_{(1 / 2)-\lambda}^{(1 / 2)+\lambda} \phi_{t}^{2}(x, t) \mathrm{d} x \mathrm{~d} t=C \int_{0}^{1} a(t) \int_{\omega} \phi_{t}^{2}(x, t) \mathrm{d} x \mathrm{~d} t
$$

which is exactly what we need to prove that the energy decays exponentially to zero in this case. This proves Theorem 2.3 in the case $q=2$ and $T=1 / 2$.

For $T \neq 1 / 2$, the two conditions are not the same. We still can deduce results of uniform exponential stabilization from Theorem 5.1, but it is not sufficient to prove Theorem 2.3.

For example, consider the case $T=1 / 4$. If we assume $\lambda>(1-T) / 2=3 / 8$, we can apply (5.8) with $T^{*}=T=1 / 4$ and we obtain

$$
E_{\phi}(0) \leq C \int_{0}^{1 / 4} \int_{(1 / 2)-\lambda}^{(1 / 2)+\lambda} \phi_{t}^{2}(x, t) \mathrm{d} x \mathrm{~d} t \leq C \int_{0}^{1} a(t) \int_{\omega} \phi_{t}^{2}(x, t) \mathrm{d} x \mathrm{~d} t,
$$

which proves that the energy decays exponentially to zero in this case. Note that the assumption " $\lambda>(1-$ $T) / 2=3 / 8$ " and this inequality correspond, as we can see in Figure 5 , to the fact that each optic ray crosses the damping region during the period $(0, T)=(0,1 / 4)$.

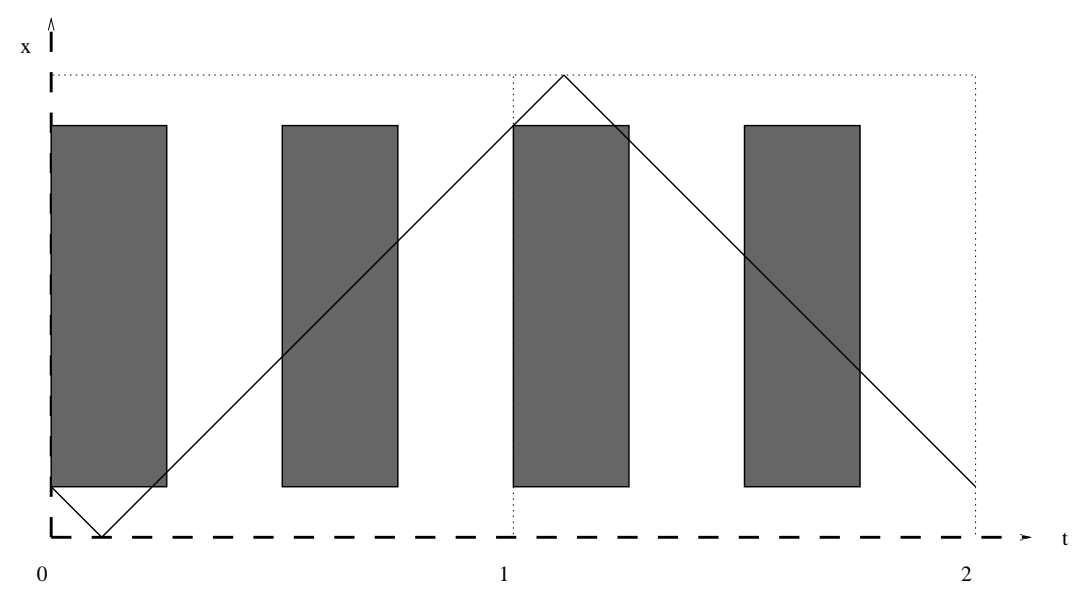

Figure 5. 
If we consider the same case $T=1 / 4$ under the assumption of Theorem 2.3 when $q=2$, i.e. with $\lambda>T / 2$ $=1 / 8$, we can still apply (5.8) but only with $T^{*}=3 / 4$, and we obtain

$$
\begin{aligned}
E_{\phi}(0) \leq & C \int_{0}^{3 / 4} \int_{(1 / 2)-\lambda}^{(1 / 2)+\lambda} \phi_{t}^{2}(x, t) \mathrm{d} x \mathrm{~d} t \\
= & C \int_{0}^{1 / 4} \int_{(1 / 2)-\lambda}^{(1 / 2)+\lambda} \phi_{t}^{2}(x, t) \mathrm{d} x \mathrm{~d} t+C \int_{1 / 4}^{1 / 2} \int_{(1 / 2)-\lambda}^{(1 / 2)+\lambda} \phi_{t}^{2}(x, t) \mathrm{d} x \mathrm{~d} t \\
& +C \int_{1 / 2}^{3 / 4} \int_{(1 / 2)-\lambda}^{(1 / 2)+\lambda} \phi_{t}^{2}(x, t) \mathrm{d} x \mathrm{~d} t \\
= & C \int_{0}^{1} a(t) \int_{\omega} \phi_{t}^{2}(x, t) \mathrm{d} x+C \int_{1 / 4}^{1 / 2} \int_{(1 / 2)-\lambda}^{(1 / 2)+\lambda} \phi_{t}^{2}(x, t) \mathrm{d} x \mathrm{~d} t
\end{aligned}
$$

but we need

$$
\begin{aligned}
E_{\phi}(0) & \leq C \int_{0}^{1} a(t) \int_{\omega} \phi_{t}^{2}(x, t) \mathrm{d} x \mathrm{~d} t \\
& =C \int_{0}^{1 / 4} \int_{(1 / 2)-\lambda}^{(1 / 2)+\lambda} \phi_{t}^{2}(x, t) \mathrm{d} x \mathrm{~d} t+C \int_{1 / 2}^{3 / 4} \int_{(1 / 2)-\lambda}^{(1 / 2)+\lambda} \phi_{t}^{2}(x, t) \mathrm{d} x \mathrm{~d} t .
\end{aligned}
$$

to solve our problem. Note that the assumption " $\lambda>1 / 8$ " and this inequality correspond, as we can see in Figure 6 , to the fact that each optic ray crosses the damping region during the period $(0,1 / 4)$ or during the period $(1 / 2,3 / 4)$.

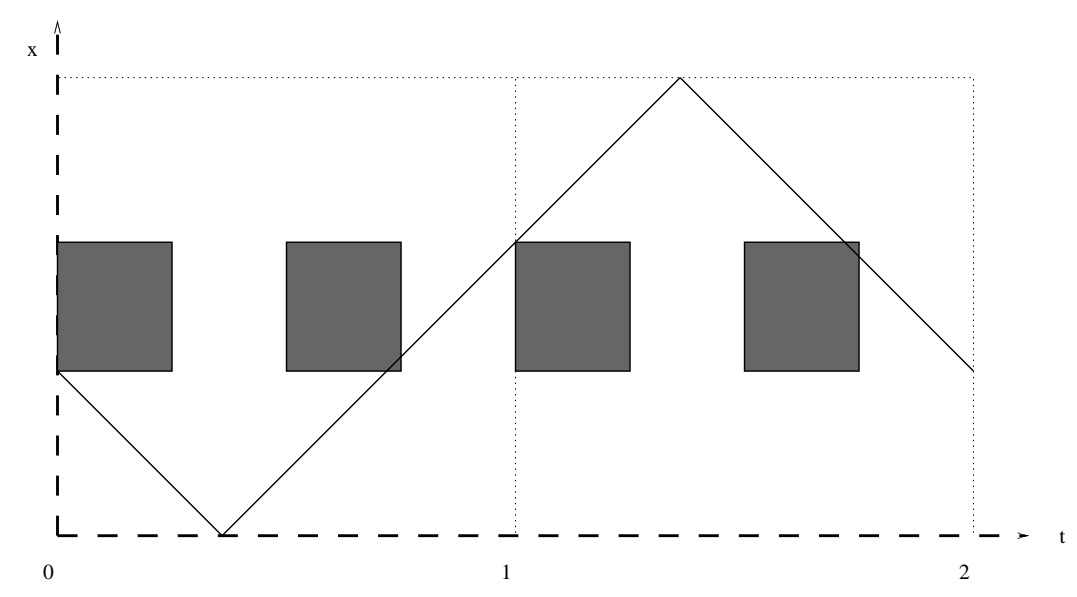

FiguRE 6.

More generally, we prove the following new "on-off" observability inequalities:

Theorem 5.2. Let $\ell \in \mathbb{N}$. Assume $T=1 /(2 \ell)$ and $T / 2<\lambda \leq 1 / 2$. Then there exists $C>0$ such that, for all $\phi$ solution of (2.6),

$$
E_{\phi}(0) \leq C \sum_{p=0}^{\ell-1} \int_{2 p T}^{(2 p+1) T} \int_{1 / 2-\lambda}^{1 / 2+\lambda} \phi_{t}^{2}(x, t) \mathrm{d} x \mathrm{~d} t=C \int_{0}^{1} a(t) \int_{\omega} \phi_{t}^{2}(x, t) \mathrm{d} x \mathrm{~d} t .
$$


Inequality (5.13) is exactly what we need to prove the exponential decay of the solutions of (1.2) when $q=2$, $1 / T \in 2 \mathbb{N}$ and $2 \lambda>T$.

Note that in the general case $(q \neq 2)$, the proof of Theorem 2.3 is based on Theorem 2.4. Theorem 2.4 is the generalization of Theorem 5.2 and its proof is similar to the proof of Theorem 5.2.

Remark. In fact repeating the arguments of the proof of Theorem 5.2, one shall prove that

$$
E_{\phi}(0) \leq C \int_{0}^{T^{*}} a(t) \int_{\omega} \phi_{t}^{2}(x, t) \mathrm{d} x \mathrm{~d} t
$$

with $T^{*}:=K q T+\rho$ at the place of $q-1$, where $K \in \mathbb{N}, \rho \in(0, T)$ and $T^{*}>1-2 \lambda$. These are the optimal conditions: indeed, one cannot expect that (5.14) holds true with $T^{*}<1-2 \lambda$ (this value is optimal when $a(t)=1$ for all $t$ ), and $T^{*}$ has necessarily to be written $K q T+\rho$ : in the other case, equation (5.14) would be satisfied with a strictly smaller value of $K$.

It is interesting to note that these results are optimal in the following sense: if $T>2 \lambda$, then we can construct some initial conditions $\left(\phi^{0}, \phi^{1}\right)$ for which $E_{\phi}(0)=1$ while

$$
\sum_{p=0}^{\ell-1} \int_{2 p T}^{(2 p+1) T} \int_{1 / 2-\lambda}^{1 / 2+\lambda} \phi_{t}^{2}(x, t) \mathrm{d} x \mathrm{~d} t=\int_{0}^{1} a(t) \int_{\omega} \phi_{t}^{2}(x, t) \mathrm{d} x \mathrm{~d} t=0 .
$$

This result correspond to the fact that, as we can see in Figure 7, there exists some optic rays that never cross the damping region at a time when the damping is effective. See Figure 7 in the case $T=1 / 4$ with $\lambda<T / 2=1 / 8$.

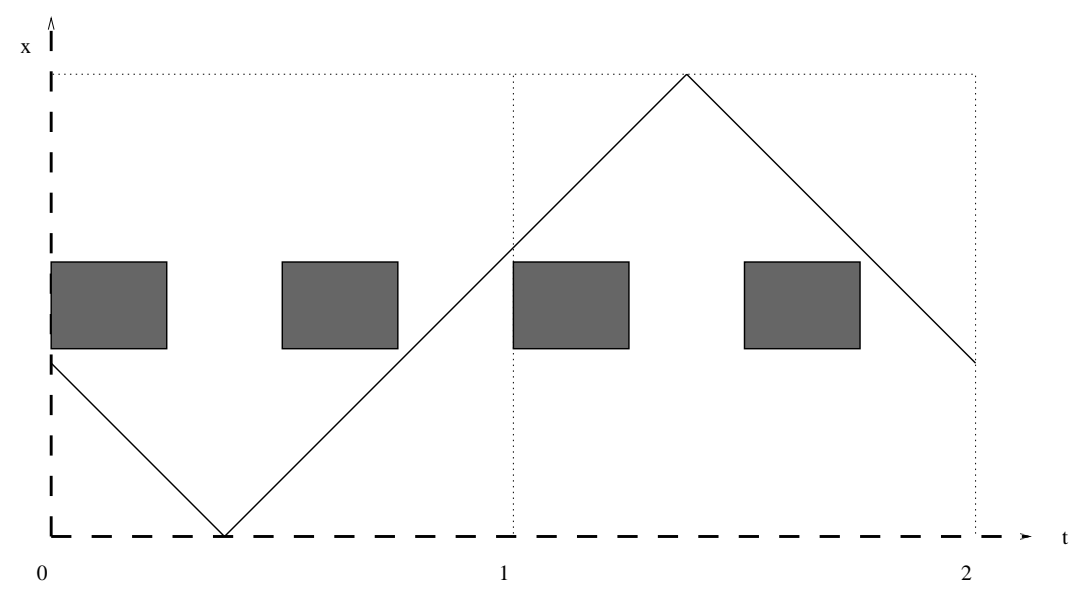

FIGURE 7.

\subsubsection{Other application of the observability inequalities: Exact controllability results}

Theorem 5.2 also obviously gives new exact controllability results, applying the method H.U.M. of J.-L. Lions [22]: given $\left(u^{0}, u^{1}\right) \in H_{0}^{1}(\Omega) \times L^{2}(\Omega)$, there exists a control $h \in L^{2}\left(\left(\cup_{p=0}^{\ell-1}(2 p T,(2 p+1) T), L^{2}(\omega)\right)\right.$ such that the solution of the problem

$$
\begin{cases}u_{t t}-u_{x x}=\chi_{\omega}(x) h(t, x), & x \in(0,1), t \geq 0, \\ u(0, t)=u(1, t)=0, & t \geq 0, \\ \left(u(x, 0), u_{t}(x, 0)\right)=\left(u^{0}(x), u^{1}(x)\right), & x \in(0,1),\end{cases}
$$


satisfies $u(1-T)=0=u_{t}(1-T)$. This implies that the control $h$ that drives the system from the state $\left(u^{0}, u^{1}\right)$ to the rest acting only on the time intervals $(2 p T,(2 p+1) T)$ for $p=0, \cdots, \ell-1$, so, roughly speaking, "only half of the time". We will generalize these results to prove that in fact we can find a control that drives the system from the state $\left(u^{0}, u^{1}\right)$ to the rest acting only on a finite number of well-chosen time intervals, whose total lenght is arbitrarily short (see [26]).

\subsubsection{Open questions}

1. It is an interesting open question to know what happens when $1 / T \in 2 \mathbb{N}$ and $2 \lambda=T$. This should be compared to the results of pointwise stabilization or pointwise controllability (see, e.g. [14]).

2. Our proof does not allow us to obtain results for nonlinear on-off feedbacks.

3. It should also be interesting to study the problem with positive negative feedbacks (1.4).

\section{Proof of Proposition 5.1 (on-off boundary feedback When $q=2$ )}

We make the proofs in the case $a_{0}=1$. Our method can easily be extended to the general cases $a_{0}>0$, in order to prove that the energy decays exponentially to zero.

\subsection{The useful formula for the energy}

Let us consider the absolutely continuous function $f:(-1,+\infty) \longrightarrow \mathbb{R}$ defined on $(-1,1)$ by:

$$
\begin{gathered}
\forall y \in(-1,0), \quad f(y):=\frac{1}{2} \int_{0}^{y} u_{x}^{0}(-z)-u^{1}(-z) \mathrm{d} z, \\
\forall y \in(0,1), \quad f(y):=\frac{1}{2} \int_{0}^{y} u_{x}^{0}(z)+u^{1}(z) \mathrm{d} z,
\end{gathered}
$$

and on $(1,+\infty)$ by induction thanks to the expression

$$
f^{\prime}(y+1)=k(y) f^{\prime}(y-1) \text { with } k(y):=\frac{a(y)-1}{a(y)+1} \text { a.e. } y \geq 0 .
$$

Note that this is equivalent to say that

$$
f^{\prime}(y+1)+f^{\prime}(y-1)=-a(y)\left(f^{\prime}(y+1)-f^{\prime}(y-1)\right) \quad \text { a.e. } y \geq 0 .
$$

Hence, using the d'Alembert's formula, we easily verify that the following expression gives a solution of the problem (1.1) (and that this solution is unique):

$$
\forall(x, t) \in(0,1) \times(0, \infty), \quad u(x, t)=f(t+x)-f(t-x) .
$$

(Relation (6.2) gives $u_{x}(1, t)=-a(t) u_{t}(1, t)$.)

We see that the energy of $u$ satisfies

$$
\forall t \geq 0, \quad E_{u}(t)=\frac{1}{2} \int_{0}^{1}\left(u_{x}^{2}(x, t)+u_{t}^{2}(x, t)\right) \mathrm{d} x=\int_{-1}^{1} f^{\prime}(t+s)^{2} \mathrm{~d} s .
$$

Now set $N \in \mathbb{N}$ and $t \in(0,2)$ such that $f^{\prime}(t-1) \in \mathbb{R}$. We deduce from (6.1) that

$$
f^{\prime}(t+2 N+1)=k(t+2 N) f^{\prime}(t+2 N-1)=\cdots=\left(\prod_{m=0}^{N} k(t+2 m)\right) f^{\prime}(t-1) .
$$


Denote

$$
K_{N}(t):=\prod_{m=0}^{N}|k(t+2 m)|
$$

Hence we get that

$$
E_{u}(2 N+2)=\int_{0}^{2} K_{N}(t)^{2} f^{\prime}(t-1)^{2} \mathrm{~d} t
$$

Note that for $t \in(0,2)$, the quantity $f^{\prime}(t-1)$ depends only on the initial conditions $\left(u^{0}, u^{1}\right)$. In the following, we will study the function $K_{N}$.

\subsection{Proof of Proposition 5.1}

Note that in this case the function $k$ satisfies:

$$
\left\{\begin{array}{l}
k(t)=0 \quad \text { if } t \in \mathcal{G}_{2}:=\bigcup_{n \in \mathbb{N}}[2 n T,(2 n+1) T) \\
k(t)=-1 \quad \text { if } t \in \mathcal{B}_{2}:=\bigcup_{n \in \mathbb{N}}[(2 n+1) T,(2 n+2) T)
\end{array}\right.
$$

Hence $K_{N}(t)=1$ if and only if $t, t+2, \cdots, t+2 N$ lie in $\mathcal{B}_{2}$, and $K_{N}(t)=0$ in the other case. ( $\mathcal{G}_{2}$ is the set of the "good" values of $t$ and $\mathcal{B}_{2}$ is the set of the "bad" values of $t$ ).

Write

$$
2=2 \ell T+r T, \text { with } \ell \in \mathbb{N} \text { and } 0 \leq r<2
$$

First assume that $r=0$ (i.e. $1 / T \in \mathbb{N})$ : this implies that $[T, 2 T) \subset[0,2) \cap \mathcal{B}_{2}$ and if $t \in \mathcal{B}_{2}$, for example $t \in[T, 2 T)$, then $t+2=t+2 \ell T \in[T+2 \ell T, 2 T+2 \ell T) \subset \mathcal{B}_{2}$, and, in the same way, $t+4, \cdots, t+2 N$ are all in $\mathcal{B}_{2}$; hence $K_{N}(t)=1$ for all $N \in \mathbb{N}$ and all $t \in \mathcal{B}_{2}$. Thus, for all $N \in \mathbb{N}$,

$$
E_{u}(2 N+2)=\int_{[0,2) \cap \mathcal{B}_{2}} K_{N}(t)^{2} f^{\prime}(t-1)^{2} \mathrm{~d} t+\int_{[0,2) \cap \mathcal{G}_{2}} K_{N}(t)^{2} f^{\prime}(t-1)^{2} \mathrm{~d} t=\int_{[0,2) \cap \mathcal{B}_{2}} f^{\prime}(t-1)^{2} \mathrm{~d} t=: E_{\infty} .
$$

Consequently, since the energy is nonincreasing and its value at times $2 N+2$ remains constant, it is constant on $[2,+\infty)$ and equal to $E_{\infty}$. This quantity depends only on the initial conditions $\left(u^{0}, u^{1}\right)$ and is non equal to zero if the initial conditions are well chosen. Note that for all $t \geq 2, E_{u}(t)=E_{\infty}$, and $E_{\infty}=0$ if and only if the function $t \mapsto f^{\prime}(t-1)$ is equal to zero on $[0,2) \cap \mathcal{B}_{2}$. In particular, if $u^{0}$ and $u^{1}$ are such that the function $f$ is compactly supported in $\left\{t: t+1 \in \mathcal{B}_{2} \cap[0,2)\right\}$, then the energy of the associated solution remains constant with time.

Now assume that $0<r \leq 1$. (Note that in this case, $d_{T}=d(1 / T, \mathbb{N})=r / 2>0$.) We claim that $K_{N}(t)=0$ if $N \geq \frac{1}{r}$. This follows from the following lemma (see the proof later):

Lemma 6.1. Fix $N \geq \frac{1}{r}$. Given $t \in[0,2)$, it is not possible for $t, t+2, \cdots, t+2 N$ to be all in $\mathcal{B}_{2}$.

In other words, Lemma 6.1 means that each optic ray touches the boundary point $x=1$ in time at most $2 N$ and at an instant time $t$ where the damping is effective. Note also that Lemma 6.1 implies that $K_{N}(t)=0$ for all $t \in[0,2)$, thus $E_{u}(2 N+2)=0$, which proves (5.1) in this case.

At last we assume that $1<r<2$. (Note that in this case, $d_{T}=1-r / 2>0$.) This case is completely analogous to the previous one. We claim that $K_{N}(t)=0$ if $N \geq \frac{1}{2-r}$. This follows from the following lemma (see the proof later): 
Lemma 6.2. Fix $N \geq \frac{1}{2-r}$. Given $t \in[0,2)$, it is not possible for $t, t+2, \cdots, t+2 N$ to be all in $\mathcal{B}_{2}$.

On the same way, it implies $K_{N}(t)=0$ for all $t \in[0,2)$ if $N(2-r) \geq 1$, and so $E_{u}(2 N+2)=0$. This proves (5.1) in this case, which ends the proof of Proposition 5.1.

Proof of Lemma 6.1. We assume that $0<r \leq 1$ and we prove the result by contradiction: assume that $t$, $t+2, \cdots, t+2 N$ are all in $\mathcal{B}_{2}$ for some $t \in[0,2)$. Then first $t \in[0,2) \cap \mathcal{B}_{2}$ : hence there exists $n_{0} \in \mathbb{N}$ such that $t \in\left[\left(2 n_{0}+1\right) T,\left(2 n_{0}+2\right) T\right)$; then using $2=2 \ell T+r T$ with $0<r T \leq T$, we deduce

$$
\begin{aligned}
t+2 \in\left[\left(2 n_{0}+1\right) T+2 \ell T,\left(2 n_{0}+2\right) T+2 \ell T+T\right) & =\left[\left(2 \ell+2 n_{0}+1\right) T,\left(2 \ell+2 n_{0}+2\right) T\right) \\
& \cup\left[\left(2 \ell+2 n_{0}+2\right) T,\left(2 \ell+2 n_{0}+2\right) T+T\right) .
\end{aligned}
$$

Note that $\left[\left(2 \ell+2 n_{0}+1\right) T,\left(2 \ell+2 n_{0}+2\right) T\right) \subset \mathcal{B}_{2}$ whereas $\left[\left(2 \ell+2 n_{0}+2\right) T,\left(2 \ell+2 n_{0}+3\right) T\right) \subset \mathcal{G}_{2}$. Since $t+2 \in \mathcal{B}_{2}$, then $t+2 \in\left[\left(2 \ell+2 n_{0}+1\right) T,\left(2 \ell+2 n_{0}+2\right) T\right)$; an easy induction argument shows that for all $m \in\{0, \cdots, N\}$

$$
t+2 m \in\left[\left(2 m \ell+2 n_{0}+1\right) T,\left(2 m \ell+2 n_{0}+2\right) T\right) .
$$

Thus we deduce (with $m=N$ and using $t \geq\left(2 n_{0}+1\right) T$ ),

$$
\left(2 n_{0}+1\right) T+2 N \leq t+2 N<\left(2 N \ell+2 n_{0}+2\right) T,
$$

which implies that

$$
r N T=N(2-2 \ell T)<T
$$

which is false.

Proof of Lemma 6.2. We assume that $1<r<2$ and we prove by contradiction that it is not possible for $t$, $t+2, \cdots, t+2 N$ to be all in $\mathcal{B}_{2}$ if $N \geq 1 /(2-r)$. Indeed, if $t, t+2, \cdots, t+2 N$ are all in $\mathcal{B}_{2}$, then

$$
t+2 N \in\left[\left(2 N(\ell+1)+2 n_{0}+1\right) T,\left(2 N(\ell+1)+2 n_{0}+2\right) T\right),
$$

hence (using $\left.t \leq\left(2 n_{0}+2\right) T\right)$,

$$
\left(2 n_{0}+1\right) T+(2 \ell+2) N T \leq t+2 N<\left(2 n_{0}+2\right) T+2 N,
$$

which implies that

$$
N(2-r)<1 .
$$

Proof of the optimality of the result. Note that our result is optimal: assume that $0<r \leq 1$ and denote $N_{T}$ the smallest integer such that $r N_{T} \geq 1$. Hence we have:

$$
\frac{1}{r} \leq N_{T}<\frac{1}{r}+1-\varepsilon
$$

for $\varepsilon$ small enough. Then it is easy to verify that if $t \in[T,(1+\varepsilon) T) \cap[0,2)$, then $t, t+2, \cdots, t+2(N-1)$ are all in $\mathcal{B}_{2}$ and so if the initial conditions are such that the corresponding function $f$ is supported in $[T-1$, $(1+\varepsilon) T-1) \cap[-1,1)$, then $E_{u}\left(2 N_{T}\right)=E_{u}(0)$, whereas $E_{u}\left(2 N_{T}+2\right)=0$.

The same phenomenom occurs if $1 \leq r<2$. 


\section{Proof of Theorem 2.1 (on-off boundary feedback in the general Case)}

Now we assume that $a=1$ on $[0, T), a=0$ on $[T, q T)$ and $a$ is $q T$-periodic. Define

$$
\left\{\begin{array}{l}
\mathcal{G}_{q}:=\bigcup_{n \in \mathbb{N}}[q n T,(q n+1) T) \\
\mathcal{B}_{q}:=\bigcup_{n \in \mathbb{N}}[(q n+1) T, q(n+1) T) .
\end{array}\right.
$$

Write

$$
2=q \ell T+r T, \text { with } \ell \in \mathbb{N} \text { and } 0 \leq r<q .
$$

\subsection{Stability if $\mathbf{T}$ is different of some exceptional values}

We prove the following:

Lemma 7.1. Assume that

$$
r \notin \mathcal{L}:=\left\{r_{0}=0\right\} \cup\left\{r_{s, p}=\frac{s}{p} q: p=2, \cdots, q-1, s=1, \cdots, p-1\right\} .
$$

Then the energy of every solution achieves zero in finite time.

Note that $r \in \mathcal{L}$ if and only if (2.1) is satisfied.

Proof of Lemma 7.1. First assume that there exists $m \geq 1$ and $k \in \mathbb{N}$ such that

$$
k q<m r \leq k q+1 \text { i.e. } m r \in(0,1] \bmod q .
$$

Then we claim that the energy of every solution achieves zero in finite time: indeed denote $\rho:=m r-k q \in(0,1]$ and choose $N$ such that $N \rho \geq q-1$; then if $t, t+2, \cdots, t+2 N m$ are all in $\mathcal{B}_{q}$, then of course $t, t+2 m$, $t+4 m, \cdots, t+2 N m$ are all in $\mathcal{B}_{q}$; but

$$
t+2 m=t+m q l T+m r T=t+\rho T+q(m l+k) T=t+\rho T \bmod q T,
$$

and thus $t, t+\rho T, t+2 \rho T, \cdots, t+N \rho T$ are all in $\mathcal{B}_{q}$, which is not possible since $\rho \in(0,1]$ and $N \rho \geq q-1$ (same proof as Lem. 6.1). Hence $E_{u}(2 m N+2)=0$.

Let us specify the real numbers $r$ that satisfy (7.3). For $r \in(0, q)$, denote $\rho^{\prime}:=\frac{r}{q} \in(0,1)$. Condition (7.3) is equivalent to say that there exists $m \geq 1$ such that

$$
\left\{m \rho^{\prime}\right\} \in\left(0, \frac{1}{q}\right]
$$

where $\{x\}$ denotes the fractional part of $x$. This is clearly true if $\rho^{\prime}:=\frac{1}{q^{\prime}}$ with $q^{\prime} \geq q$ (and $m:=1$ ); this is also true if $\rho^{\prime}:=\frac{p^{\prime}}{q^{\prime}}$ with $q^{\prime} \geq q$ and $\operatorname{pgcd}\left(p^{\prime}, q^{\prime}\right)=1$ : indeed, there exists $\left(p^{\prime \prime}, q^{\prime \prime}\right)$ such that $p^{\prime \prime} p^{\prime}-q^{\prime \prime} q^{\prime}=1$, and then $p^{\prime \prime} \rho^{\prime}=\frac{1}{q^{\prime}}+q^{\prime \prime}$; at last (7.4) is also true if $\rho^{\prime} \notin \mathbb{Q}$, since in that case the set $\left\{\left\{m \rho^{\prime}\right\}, m \in \mathbb{N}\right\}$ is everywhere dense in $[0,1)$. Thus if $r \notin \mathcal{L}$, equation (7.3) is satisfied, and the proof of Lemma 7.1 is complete.

\subsection{Non stability if $\mathbf{T}$ is equal to one of those exceptional values}

We prove that if $r \in \mathcal{L}$, then we have no more strong stability property.

First, for all $q \geq 2$, the result is clear for the value $r=r_{0}=0$. Indeed in this case we have $2=q \ell T$. It implies that: $\left(t \in \mathcal{B}_{q}\right) \Longrightarrow\left(t+2 \in \mathcal{B}_{q}\right)$. Thus it is sufficient to prove that there exists a non empty interval $I \subset\left[0,2\left[\cap \mathcal{B}_{q}\right.\right.$, which is clear since $T<2$. 
Next we assume that $q \geq 3$ and we prove this result for all the values $r=r_{s, p}=s q / p$ with $p \in\{2, \ldots, q-1\}$ and $s \in\{1, \ldots, p-1\}$. Indeed in this case we have

$$
2=q \ell T+\frac{s}{p} q T
$$

Note that it implies that if $t \in \mathcal{B}_{q}$, then $t+2 p \in \mathcal{B}_{q}$. Thus, if we set

$$
\mathcal{E}=\left\{t \in \left[0,2\left[: t, t+2, t+4, \ldots, t+2(p-1) \in \mathcal{B}_{q}\right\},\right.\right.
$$

it is sufficient to prove that there exists a non empty interval $I \subset \mathcal{E}$.

Let $I=[T, q T / p[$. We verify that $I \neq \emptyset($ since $q / p>1)$ and $I \subset[0,2)$ (since $T \leq 2 p / s q \leq 2 p / q$ from (7.5)). Moreover $I \subset \mathcal{B}_{q}$ (indeed $I \subset[T, q T)$ ).

It remains to prove that $\tau_{2 k}(I) \subset \mathcal{B}_{q}$ for all $k \in\{1,2, \ldots, p-1\}$, where $\tau_{2 k}$ denotes the translation of $2 k$. Fix $k \in\{1,2, \ldots, p-1\}$. There exist $n_{1}, n_{2} \in \mathbb{N}$ such that

$$
s k=n_{1} p+n_{2} \quad \text { with } 0 \leq n_{2} \leq p-1 .
$$

Then using $(7.5,7.6)$ and using $n_{2}+1 \leq p$, we obtain

$$
\begin{aligned}
\tau_{2 k}(I) & =\left[T+2 k, \frac{1}{p} q T+2 k\right)=\left[T+q k \ell T+\frac{s k}{p} q T, \frac{1}{p} q T+q k \ell T+\frac{s k}{p} q T\right) \\
& =\left[\left(k \ell+n_{1}\right) q T+T+\frac{n_{2}}{p} q T,\left(k \ell+n_{1}\right) q T+\frac{n_{2}+1}{p} q T\right) \\
& \subset\left[\left(k \ell+n_{1}\right) q T+T,\left(k \ell+n_{1}\right) q T+q T\right) \subset \mathcal{B}_{q},
\end{aligned}
$$

which proves the result.

\section{Proof of Theorem 2.2 (Positive-negative Boundary feedbacks)}

It is easy to see that now, with the notations used in the previous sections, we have

$$
E_{u}(2 N+2)=\int_{0}^{2} K_{N}(t)^{2} f^{\prime}(t-1)^{2} \mathrm{~d} t
$$

with

$$
K_{N}(t)=\left|\frac{a_{0}-1}{a_{0}+1}\right|^{P_{N}(t)}\left|\frac{b_{0}+1}{b_{0}-1}\right|^{N+1-P_{N}(t)},
$$

where $P_{N}(t)$ is equal to the number of $m \in\{0, \cdots, N\}$ such that $t+2 m \in \mathcal{G}_{2}$. If $T$ is exceptional, then we can find initial conditions such that $P_{N}(t)=0$ for all $t$ and all $N$, hence the energy of such solutions grows exponentially to infinity. If $T$ is not exceptional i.e. if $1 / T \notin \mathbb{N}$, we have seen that there exists $N_{T}$ such that for all $t$, there exists at least one $m_{t} \in\left\{0, \cdots, N_{T}\right\}$ such that $t+2 m_{t} \in \mathcal{G}_{2}$, hence $P_{N_{T}}(t) \geq 1$. We deduce that

$$
E_{u}\left(2 N_{T}+2\right) \leq E_{u}(0)\left|\frac{a_{0}-1}{a_{0}+1}\right|\left|\frac{b_{0}+1}{b_{0}-1}\right|^{N_{T}} .
$$

Obviously, this gives a sufficient condition on the exponential decay, but it is far from being optimal. Indeed, we proved that we can find initial conditions such that $P_{N_{T}}(t)=1$, but then nothing tells that $P_{2 N_{T}}(t)=2$, and in fact this is wrong in general. So we have to study carefully the function $P_{N}$, and obtain asymptotic uniform (in $t$ ) estimates as $N \rightarrow \infty$. More precisely we have to study the ratio $P_{N}(t) /(N+1)$ and its limit as $N \rightarrow \infty$. 


\subsection{The cyclic case: $1 / T \in \mathbb{Q} \backslash \mathbb{N}$}

Let $1 / T=p^{\prime} / q^{\prime}$ where $p^{\prime}$ and $q^{\prime}$ are relatively prime integers. We have to study the numbers of integers $m$ such that

$$
\left\{\frac{1}{2 T}(t+2 m)\right\}=\left\{\frac{t}{2 T}+\frac{m}{T}\right\}=\left\{t^{\prime}+\frac{m p^{\prime}}{q^{\prime}}\right\} \in\left[0, \frac{1}{2}\right)
$$

where $t^{\prime}=t /(2 T)$. Note that

$$
\left\{t^{\prime}+\frac{m p^{\prime}}{q^{\prime}}\right\}=\left\{t^{\prime}+\left\{\frac{m p^{\prime}}{q^{\prime}}\right\}\right\}
$$

Since

$$
\left\{\left\{\frac{m p^{\prime}}{q^{\prime}}\right\}, m \in \mathbb{N}\right\}=\left\{\left\{\frac{m p^{\prime}}{q^{\prime}}\right\}, m \in\left\{0, \cdots, q^{\prime}-1\right\}\right\}=\left\{0, \frac{1}{q^{\prime}}, \frac{2}{q^{\prime}}, \cdots, \frac{q^{\prime}-1}{q^{\prime}}\right\},
$$

it is sufficient to count the number of the $q^{\prime}$ terms

$$
\left\{t^{\prime}\right\},\left\{t^{\prime}+\frac{1}{q^{\prime}}\right\},\left\{t^{\prime}+\frac{2}{q^{\prime}}\right\}, \cdots,\left\{t^{\prime}+\frac{q^{\prime}-1}{q^{\prime}}\right\}
$$

that are in $\left[0, \frac{1}{2}\right)$. Since we have $q^{\prime}$ real numbers separated by the distance $1 / q^{\prime}$, we have exactly $q^{\prime} / 2$ elements in $\left[0, \frac{1}{2}\right)$ if $q^{\prime}$ is even, and $\left(q^{\prime}-1\right) / 2$ or $\left(q^{\prime}+1\right) / 2$ elements in $\left[0, \frac{1}{2}\right)$ if $q^{\prime}$ is odd. Then given $N$, it is sufficient to write $N=q^{\prime} \nu+r^{\prime}$ with $0 \leq r^{\prime}<q^{\prime}$ to get estimates of $P_{N}(t)$.

If $q^{\prime}$ is even, we get the estimates

$$
\nu \frac{q^{\prime}}{2} \leq P_{N}(t) \leq(\nu+1) \frac{q^{\prime}}{2}
$$

Hence

$$
E_{u}(0)\left|\frac{a_{0}-1}{a_{0}+1}\right|^{(\nu+1) q^{\prime}}\left|\frac{b_{0}+1}{b_{0}-1}\right|^{2(N+1)-(\nu+1) q^{\prime}} \leq E_{u}(2 N+2) \leq E_{u}(0)\left|\frac{a_{0}-1}{a_{0}+1}\right|^{\nu q^{\prime}}\left|\frac{b_{0}+1}{b_{0}-1}\right|^{2(N+1)-\nu q^{\prime}},
$$

and the behavior of the energy at infinity depends on

$$
K_{T}:=\left|\frac{a_{0}-1}{a_{0}+1}\right|^{1 / 2}\left|\frac{b_{0}+1}{b_{0}-1}\right|^{1 / 2}:
$$

if $K_{T}<1$, the energy goes to 0 as $K_{T}^{t}$; if $K_{T}>1$, then the energy goes to infinity as $K_{T}^{t}$.

If $q^{\prime}$ is odd, we get the estimate

$$
\nu \frac{q^{\prime}-1}{2} \leq P_{N}(t) \leq(\nu+1) \frac{q^{\prime}+1}{2}
$$

Hence

$$
\begin{gathered}
\nu \frac{q^{\prime}}{2} \leq P_{N}(t) \leq(\nu+1) \frac{q^{\prime}}{2} . \\
E_{u}(0)\left|\frac{a_{0}-1}{a_{0}+1}\right|^{(\nu+1)\left(q^{\prime}+1\right)}\left|\frac{b_{0}+1}{b_{0}-1}\right|^{2(N+1)-(\nu+1)\left(q^{\prime}+1\right)} \leq E_{u}(2 N+2) \leq E_{u}(0)\left|\frac{a_{0}-1}{a_{0}+1}\right|^{\nu\left(q^{\prime}-1\right)}\left|\frac{b_{0}+1}{b_{0}-1}\right|^{2(N+1)-\nu\left(q^{\prime}-1\right)},
\end{gathered}
$$


and the behavior of the energy at infinity depends on

$$
K_{T}:=\left|\frac{a_{0}-1}{a_{0}+1}\right|^{\left(q^{\prime}-1\right) / 2 q^{\prime}}\left|\frac{b_{0}+1}{b_{0}-1}\right|^{1-\left(q^{\prime}-1\right) / 2 q^{\prime}}:
$$

if $K_{T}<1$, the energy goes to 0 as $K_{T}^{t}$; if $K_{0}>1$, then we can always find initial conditions (the "worst initial conditions") such that the energy behaves exactly as $K_{T}^{t}$.

\subsection{The equidistributed case: $1 / T \notin \mathbb{Q}$}

When $\theta:=1 / T \notin \mathbb{Q}$, using a consequence of a theorem of Weyl (see, e.g. [28]), we know that the set $\left\{\left\{t^{\prime}+m \theta\right\}, m \in \mathbb{N}\right\}$ is uniformly distributed in $[0,1)$. Hence $P_{N}(t) \sim(N+1) / 2$ as $N \rightarrow \infty$. However we need some uniformity in $t$ to conclude.

In fact, using the same ideas contained in the proof of the consequence of Weyl's theorem, we will prove that the sequence of functions $t \mapsto P_{N}(t) /(N+1)-1 / 2$ converges to 0 uniformly in $t \in[0,2]$ as $N \rightarrow \infty$.

Indeed, we will prove the following:

Lemma 8.1. For all continuous 1-periodic function,

$$
S_{n}\left(f_{t}\right) \rightarrow \int_{0}^{1} f_{t}(x) \mathrm{d} x=\int_{0}^{1} f(x) \mathrm{d} x \quad \text { as } n \rightarrow \infty
$$

uniformly with respect to $t \in \mathbb{R}$, where we denote $f_{t}(x):=f(x+t)$ and

$$
S_{n}(f):=\frac{1}{n+1} \sum_{k=0}^{n} f(k \theta)
$$

Then it is easy to conclude: denote $\chi$ the 1 -periodic function equal to 1 on $[0,1 / 2)$ and 0 on $[1 / 2,1)$, and $\chi(t)(x)=\chi(x+t)$. Then we are interested in studying

$$
P_{N}(t)=\sum_{k=0}^{N} \chi\left(\frac{t}{2 T}+\frac{k}{T}\right)=(N+1) S_{N}\left(\chi_{t / 2 T}\right) .
$$

Choose $\varepsilon>0$ and denote $f_{\varepsilon}$ and $F_{\varepsilon}$ two continuous and 1-periodic functions such that

$$
\begin{gathered}
0 \leq f_{\varepsilon} \leq \chi \leq F_{\varepsilon}, \\
\int_{0}^{1} F_{\varepsilon}(x)-f_{\varepsilon}(x) \mathrm{d} x \leq \varepsilon .
\end{gathered}
$$

Then

$$
(N+1) S_{N}\left(f_{\varepsilon, t / 2 T}\right) \leq P_{N}(t) \leq(N+1) S_{N}\left(F_{\varepsilon, t / 2 T}\right) .
$$

Hence

$$
\begin{aligned}
\left|\frac{P_{N}(t)}{N+1}-\int_{0}^{1} \chi(x) \mathrm{d} x\right| & \leq\left|S_{N}\left(F_{\varepsilon, t / 2 T}\right)-\int_{0}^{1} \chi(x) \mathrm{d} x\right|+\left|S_{N}\left(f_{\varepsilon, t / 2 T}\right)-\int_{0}^{1} \chi(x) \mathrm{d} x\right| \\
& \leq\left|S_{N}\left(F_{\varepsilon, t / 2 T}\right)-\int_{0}^{1} F_{\varepsilon}(x) \mathrm{d} x\right|+\left|S_{N}\left(f_{\varepsilon, t / 2 T}\right)-\int_{0}^{1} f_{\varepsilon}(x) \mathrm{d} x\right|+2 \varepsilon \leq 4 \varepsilon
\end{aligned}
$$

using Lemma 8.1 and choosing $N$ large enough (independent of $t$ ). 
Hence in the case $q=2, P_{N}(t) /(N+1) \rightarrow 1 / 2$ as $N \rightarrow \infty$ and uniformly with respect to $t$. Hence the energy behaves as

$$
\left(\left|\frac{a_{0}-1}{a_{0}+1}\right|^{1 / 2}\left|\frac{b_{0}+1}{b_{0}-1}\right|^{1 / 2}\right)^{t}
$$

Proof of Lemma 8.1. Let $f: \mathbb{R} \rightarrow \mathbb{R}$ continuous and 1-periodic. Let

$$
Q: x \mapsto \sum_{m=0}^{M} c_{m} \mathrm{e}^{2 i \pi m x}
$$

be a trigonometrical polynomial function close to $f$ in the space of the continuous bounded functions endowed with the usual $\|\cdot\|_{\infty}$. Denote $f_{t}(x):=f(x+t)$ and $Q_{t}(x):=Q(x+t)$, and

$$
S_{n}(f):=\frac{1}{n+1} \sum_{k=0}^{n} f(k \theta) .
$$

First we verify that property for the polynomial function $Q$ : since

$$
\begin{aligned}
Q_{t}(x)=\sum_{m=0}^{M} c_{m} \mathrm{e}^{2 i \pi m(x+t)} & =\sum_{m=0}^{M} c_{m} \mathrm{e}^{2 i \pi m t} \mathrm{e}^{2 i \pi m x} \\
& =\int_{0}^{1} Q_{t}(x) \mathrm{d} x+\sum_{m=1}^{M} c_{m} \mathrm{e}^{2 i \pi m t} \mathrm{e}^{2 i \pi m x}
\end{aligned}
$$

we easily see that

$$
\begin{aligned}
\left|S_{n}\left(Q_{t}\right)-\int_{0}^{1} Q_{t}(x) \mathrm{d} x\right| & =\left|\frac{1}{n+1} \sum_{k=0}^{n} \sum_{m=1}^{M} c_{m} \mathrm{e}^{2 i \pi m t} \mathrm{e}^{2 i \pi m k \theta}\right| \\
& \leq \frac{1}{n+1} \sum_{m=1}^{M}\left|c_{m}\right|\left|\sum_{k=0}^{n} \mathrm{e}^{2 i \pi m k \theta}\right| \\
& \leq \frac{1}{n+1} \sum_{m=1}^{M}\left|c_{m}\right|\left|\frac{2}{1-\mathrm{e}^{2 i \pi m \theta}}\right| \leq \frac{c(Q)}{n+1}
\end{aligned}
$$

where $c(Q)$ is some constant that depends on $Q$ but not on $t$.

Next we conclude the proof of Lemma 8.1:

$$
\begin{aligned}
\left|S_{n}\left(f_{t}\right)-\int_{0}^{1} f_{t}(x) \mathrm{d} x\right| & \leq\left|S_{n}\left(f_{t}\right)-S_{n}\left(Q_{t}\right)\right|+\left|S_{n}\left(Q_{t}\right)-\int_{0}^{1} Q_{t}(x) \mathrm{d} x\right|+\left|\int_{0}^{1} Q_{t}(x) \mathrm{d} x-\int_{0}^{1} f_{t}(x) \mathrm{d} x\right| \\
& \leq 2\|f-Q\|_{\infty}+\frac{c(Q)}{n+1} .
\end{aligned}
$$


Hence, given $\varepsilon>0$, there exists some polynomial function $Q_{\varepsilon}$ and $n_{\varepsilon}$ such that for all $n \geq n_{\varepsilon}$ we have

$$
\left|S_{n}\left(f_{t}\right)-\int_{0}^{1} f_{t}(x) \mathrm{d} x\right| \leq 2\left\|f-Q_{\varepsilon}\right\|_{\infty}+\frac{c\left(Q_{\varepsilon}\right)}{n+1} \leq 3 \varepsilon
$$

for all $t \in \mathbb{R}$.

\section{Proof of Proposition 5.2 (estimate of the extinction time)}

Proof of (ii). When $T$ is not an exceptional value, the proof of Theorem 2.1 provides an estimate of the time in which the energy achieves zero: we proved that $E_{u}(2 m N+2)=0$ if $m$ and $N$ are integers chosen such that

$$
0<\left\{m\left\{\frac{2}{q T}\right\}\right\} \leq \frac{1}{q} \text { and } N\left\{m\left\{\frac{2}{q T}\right\}\right\} \geq \frac{q-1}{q}
$$

which proves (ii).

Remark. Note that if $q=2$, we can retrieve the result of Proposition 5.1. Indeed if $q=2$, equation (9.1) means that

$$
0<\left\{m \frac{r}{2}\right\} \leq \frac{1}{2} \text { and } N \rho \geq 1
$$

where $2=2 \ell T+r T$ with $0<r<2$ and $\rho:=\{m r\}$. Thus in particular if $0<r<1$, then (9.2) is satisfied with $m:=1$ and $N$ the smallest integer such that $N r \geq 1$, so we find again the result proved after Lemma 6.1. However, in the case $1<r<2$, we do not retrieve directly the optimal estimates proved in Lemma 6.1. Indeed, when $r=1+\varepsilon$ with $\varepsilon>0$ and small, equation (9.2) gives a really bad estimate: it is satisfied for $m=2$ and $N$ has to be large enough such that $2 N \varepsilon \geq 1$. However we can overcome this difficulty with an argument of symmetry:

Lemma 9.1. Assume that $q \geq 2$ and that $T$ and $T^{\prime}$ satisfy

$$
\left\{\frac{2}{q T^{\prime}}\right\}=1-\left\{\frac{2}{q T}\right\}
$$

Denote

$$
\mathcal{B}_{q}(T):=\bigcup_{n \in \mathbb{N}}[(q n+1) T, q(n+1) T)
$$

and

$$
\mathcal{B}_{q}\left(T^{\prime}\right):=\bigcup_{n \in \mathbb{N}}\left[(q n+1) T^{\prime}, q(n+1) T^{\prime}\right)
$$

Fix $N \in \mathbb{N}$. Then if $t, t+2, \cdots, t+2 N$ are all in $\mathcal{B}_{q}(T)$, then $t^{\prime}, t^{\prime}+2, \cdots, t^{\prime}+2 N$ are all in $\mathcal{B}_{q}\left(T^{\prime}\right)$, with

$$
t^{\prime}:=\left(\frac{t+2 N}{T}\right) T^{\prime}
$$


Note that (9.3) means that

$$
2=q \ell T+r T=q \ell^{\prime} T^{\prime}+r^{\prime} T^{\prime}
$$

with $r^{\prime}=q-r$.

Hence returning to the case $q=2$ with $1<r<2$, we deduce from Lemma 9.1 that for all $t, t, t+2, \cdots, t+2 N$ cannot be all in $\mathcal{B}_{2}$ if $N r^{\prime}=N(2-r) \geq 1$.

Remark. In a general way, if $r \in(0,1]$, Lemma 7.1 gives us the optimal estimate on $N$ : choosing $m=1$, $k=0$, we obtain that $E_{u}(2 N+2)=0$ if $N r \geq q-1$, and Lemma 9.1 allows us to say that if $q-1 \leq r<q$, then $E_{u}(2 N+2)=0$ if $N(q-r) \geq q-1$, and it is easy to see that these values of $N$ are the best possible (as in the case $q=2$ ).

Proof of Lemma 9.1. First note that $t \in \mathcal{B}_{q}(T)$ implies that $t^{\prime} \in \mathcal{B}_{q}\left(T^{\prime}\right)$. Next note that

$$
\frac{t^{\prime}+2}{T^{\prime}}=\frac{t+2 N}{T}+q \ell^{\prime}+r^{\prime}=\frac{t+2 N}{T}+q \ell^{\prime}+q-\frac{2}{T}+q \ell=\frac{t+2(N-1)}{T}+q\left(1+\ell+\ell^{\prime}\right),
$$

and we easily verify that for all $m \in\{0, \cdots, N\}$ we have

$$
\frac{t^{\prime}+2 m}{T^{\prime}}=\frac{t+2(N-m)}{T}+m q\left(1+\ell+\ell^{\prime}\right)
$$

Hence $t+2(N-m) \in \mathcal{B}_{q}(T)$ implies that $t^{\prime}+2 m \in \mathcal{B}_{q}\left(T^{\prime}\right)$.

Proof of $(i)$. Now we study carefully the case $q=3$ and we prove (i). Write

$$
2=3 \ell T+r T, \text { with } \ell \in \mathbb{N} \text { and } 0<r<3:
$$

Theorem 2.1 implies that the energy achieves zero in finite time if $r$ is different from 0 and $3 / 2$. When $0<r \leq 1$ and when $2 \leq r<3$ (i.e. when we are in the case (5.3)), we already know the best possible estimate. It remains to study the case $1<r<2$ or only the case $\frac{3}{2}<r<2$ using Lemma 9.1. Note that we are in the case (5.4). First we prove the following:

Lemma 9.2. Assume that $\frac{3}{2}<r<2$. Fix $N^{\prime} \geq \frac{1}{2 r-3}$ and choose $N:=2 N^{\prime}$. Given $t \in[0,2)$, it is not possible for $t, t+2, \cdots, t+2 N$ to be all in $\mathcal{B}_{3}$.

Note that Lemma 9.2 implies that the energy achieves zero in time at most $2 N+2$, but is weaker than (5.5).

Proof of Lemma 9.2. Assume that $3 / 2<r<2$. Assume that $t, t+2, \cdots, t+2 N=t+4 N^{\prime}$ are all in $\mathcal{B}_{3}$ and assume that $t \in\left[\left(3 n_{0}+1\right) T,\left(3 n_{0}+3\right) T\right)$. It is interesting to distinguish the two cases $t \in\left[\left(3 n_{0}+1\right) T,\left(3 n_{0}+2\right) T\right)$ and $t \in\left[\left(3 n_{0}+2\right) T,\left(3 n_{0}+3\right) T\right)$.

Assume first that $t \in\left[\left(3 n_{0}+1\right) T,\left(3 n_{0}+2\right) T\right)$. Then, using that $2=3 \ell T+r T$ with $T<r T<2 T$,

$$
t+2 \in\left[\left(3 n_{0}+1\right) T+3 \ell T+T,\left(3 n_{0}+2\right) T+3 \ell T+2 T\right) .
$$

Since $t+2$ is in $\mathcal{B}_{3}$, this gives that

$$
t+2 \in\left[3 n_{0} T+3 \ell T+2 T, 3 n_{0} T+3 \ell T+3 T\right) .
$$

Using that $t+4$ is in $\mathcal{B}_{3}$, we obtain

$$
t+4 \in\left[3 n_{0} T+3 T+6 \ell T+T, 3 n_{0} T+3 T+6 \ell T+2 T\right) .
$$


Then we can easily prove by induction that for all $m \in\left\{0, \cdots, N^{\prime}\right\}$ we have

$$
t+4 m \in\left[3 n_{0} T+3 m T+6 m \ell T+T, 3 n_{0} T+3 m T+6 m \ell T+2 T\right) .
$$

Thus, for all $m \in\left\{0, \cdots, N^{\prime}\right\}$ we have

$$
3 n_{0} T+T \leq t+(2 r-3) m T<3 n_{0} T+2 T,
$$

and for all $m \in\left\{0, \cdots, N^{\prime}-1\right\}$ we have

$$
3\left(n_{0}+\ell\right) T+2 T \leq t+2+(2 r-3) m T<3\left(n_{0}+\ell\right) T+3 T .
$$

Assume that $\frac{3}{2}<r<2$. Since $t \geq 3\left(n_{0}+1\right) T$, we easily derive from (9.5) that

$$
3 n_{0} T+T+(2 r-3) N^{\prime} T<3 n_{0} T+2 T,
$$

which contradicts our choice of $N^{\prime}$. When $1<r<\frac{3}{2}$, the reasonment is identical. And then we can treat similarily the case $t \in\left[\left(3 n_{0}+2\right) T,\left(3 n_{0}+3\right) T\right)$.

However we did not use all the informations we had: we only used the fact that $t, t+4, \cdots, t+4 N^{\prime}$ were in $\mathcal{B}_{3}$. Now we prove the following optimal result:

Lemma 9.3. (i) Let $N_{T}^{\prime}$ be defined (5.5) and $N_{T}:=2 N_{T}^{\prime}-1$ Then given $t \in[0,2)$, it is not possible for $t$, $t+2, \cdots, t+2 N_{T}$ to be all in $\mathcal{B}_{3}$.

Proof of (ii). Moreover there exists a non empty interval $I \subset[0,2)$ such that $\tau_{2 k}(I) \subset \mathcal{B}_{3}$ for all $k \in\{0, \cdots$, $\left.N_{T}-1\right\}$, where $\tau_{2 k}$ denotes the translation of $2 k$.

Note that Lemma 9.3 implies that if you choose initial conditions supported in $I$, then $E_{u}\left(2 N_{T}\right)=E_{u}(0)$ whereas for every solution $v, E_{v}\left(2 N_{T}+2\right)=0$.

Proof of Lemma 9.3. (i) It is based on (9.5) and (9.6). Assume that $3 / 2<r<2$. Assume that $t, t+2, \cdots$, $t+4 N^{\prime}-2$ are all in $\mathcal{B}_{3}$. Note that it implies that $t+4 N^{\prime}$ is also in $\mathcal{B}_{3}$ (indeed if $t^{\prime} \in \mathcal{B}_{3}$ and $t^{\prime}+2 \in \mathcal{B}_{3}$, we can deduce, using $3 / 2<r<2$, that $t^{\prime}+4 \in \mathcal{B}_{3}$ ). Then we derive from (9.5) and (9.6) that

$$
t+(2 r-3) N^{\prime} T<3 n_{0} T+2 T,
$$

and

$$
t+2+(2 r-3)\left(N^{\prime}-1\right) T<3\left(n_{0}+\ell\right) T+3 T .
$$

But we easily verify that (9.8) implies (9.7) since $r<2$. Then, using $t \geq 3\left(n_{0}+1\right) T$ and $2=3 l T+r T$, we deduce from (9.8) that

$$
3 n_{0} T+T+(2 r-3)\left(N^{\prime}-1\right) T<3 n_{0} T+(3-r) T,
$$

thus $(2 r-3) N^{\prime}<r-1$. But $N_{T}^{\prime}$ (defined by (5.5)) is the smallest integer such that

$$
(2 r-3) N_{T}^{\prime} \geq r-1 .
$$

Hence it is not possible for $t, t+2, \cdots, t+4 N_{T}^{\prime}-2$ to be all in $\mathcal{B}_{3}$. 
(ii) On the other hand, it is possible to find a small interval $I \subset \mathcal{B}_{3} \cap[0,2)$ such that for all $t \in I, t, t+2, \cdots$, $t+4 N_{T}^{\prime}-4=t+2\left(N_{T}-1\right)$ are all in $\mathcal{B}_{3}$. Indeed consider $t^{\prime}:=\left(1+\varepsilon^{\prime}\right) T$, with $\varepsilon^{\prime}>0$ (and small). Then (9.8) is satisfied for $N^{\prime}:=N_{T}^{\prime}-1$ :

$$
\begin{aligned}
T+2+(2 r-3)\left(N^{\prime}-1\right) T & =T+3 \ell T+r T+(2 r-3)\left(N_{T}^{\prime}-2\right) T<3 \ell T+(1+r+r-1-2 r+3) T \\
& =3 \ell T+3 T
\end{aligned}
$$

hence

$$
\left(1+\varepsilon^{\prime}\right) T+2+(2 r-3)\left(N_{T}^{\prime}-2\right) T<3 \ell T+3 T
$$

if $\varepsilon^{\prime}$ is small enough. Moreover

$$
\left(1+\varepsilon^{\prime}\right) T+2=3 \ell T+\left(r+1+\varepsilon^{\prime}\right) T \geq 3 \ell T+2 T
$$

Thus, for $\varepsilon^{\prime}$ small enough, we have

$$
\left\{\begin{array}{l}
3 \ell T+2 T \leq t^{\prime}+2<t^{\prime}+2+(2 r-3)\left(N_{T}^{\prime}-2\right) T<3 \ell T+3 T \\
T \leq t^{\prime}<t^{\prime}+(2 r-3)\left(N_{T}^{\prime}-1\right) T<2 T
\end{array}\right.
$$

hence we deduce from $(9.5-9.6)$ that $t^{\prime}, t^{\prime}+2, \cdots, t^{\prime}+4\left(N_{T}^{\prime}-1\right)$ are all in $\mathcal{B}_{3}$.

\section{Proof of Theorem 2.3 When $q=2$ (LOCAlly Distributed On-OFF FeEdBACK)}

The previous proofs were based on the d'Alembert's formula, which is not valid for the solutions of (1.2). Hence we will need the following useful caracterization of uniform exponential decay of the energy by an observability inequality. This generalizes the classical caracterization known in the case of time independent feebacks (see Haraux [8]). Since it is completely general, we set it in a general case.

\subsection{The useful tool}

Let $\Omega$ be an open bounded domain of $\mathbb{R}^{N}$ and $\omega$ an open subset of $\Omega$. Consider the problems

$$
\begin{gathered}
\left\{\begin{array}{l}
u_{t t}-\Delta u=-a(t) \chi_{\omega}(x) u_{t} \text { on } \Omega \times \mathbb{R}_{+}, \\
u=0 \text { on } \partial \Omega \times \mathbb{R}_{+} \\
u(0)=u^{0}, u_{t}(0)=u^{1}
\end{array}\right. \\
\left\{\begin{array}{l}
\phi_{t t}-\Delta \phi=0 \text { on } \Omega \times \mathbb{R}_{+} \\
\phi=0 \text { on } \partial \Omega \times \mathbb{R}_{+} \\
\phi(0)=\phi_{0}, \phi_{t}(0)=\phi_{1}
\end{array}\right.
\end{gathered}
$$

Then:

Proposition 10.1. Assume that the function $a: \mathbb{R}_{+} \rightarrow \mathbb{R}_{+}$is bounded and periodic of period $\tilde{T}$. Then there exists $T^{*}=K^{*} \tilde{T}$ for some $K^{*} \in \mathbb{N}$ and $C>0$ such that for all $\left(\phi_{0}, \phi_{1}\right) \in H_{0}^{1}(\Omega) \times L^{2}(\Omega)$

$$
E_{\phi}(0) \leq C \int_{0}^{T^{*}} \int_{\omega} a(t) \phi_{t}(x, t)^{2} \mathrm{~d} x \mathrm{~d} t
$$


if and only if the solutions of (10.1) decay uniformly exponentially to 0 , that means if there exists $T_{1}>0$ and $\delta>0$ such that for all $\left(u^{0}, u^{1}\right) \in H_{0}^{1}(\Omega) \times L^{2}(\Omega)$, we have

$$
\forall t>0, \quad E_{u}(t) \leq E_{u}(0) \mathrm{e}^{\delta\left(T_{1}-t\right)}
$$

This property is well known in the case of time independant feedbacks (i.e. when the function $a$ remains constant with time) and the proof is easily adaptable in the case where $a$ is a time periodic function. To be complete, we give the proof of Proposition 10.1 in Section 13.

Now consider again $\Omega=(0,1)$ and the function $a: \mathbb{R}_{+} \rightarrow \mathbb{R}_{+}$defined by (1.3). It is natural to look first to the uniformly distributed case before studying the locally distributed case.

\subsection{Proof of Theorem 2.3 when $q=\mathbf{2}$ and $\boldsymbol{\omega}=(\mathbf{0}, \mathbf{1})$}

In this section we consider the case where the feedback is uniformly distributed in $(0,1)$. Using Proposition 10.1, to prove the uniform exponential decay of the solutions of (1.2), it is sufficient to prove that the solutions of (2.6) satisfy

$$
E_{\phi}(0) \leq C \int_{0}^{T^{*}} \int_{\Omega} a(t) \phi_{t}(x, t)^{2} \mathrm{~d} x \mathrm{~d} t
$$

for some $T^{*}>0$. Equation (10.5) follows from 5.1, but there is also a simple direct proof based on spectral decomposition that we recall here: usual computations give that all the solutions of (2.6) are

$$
\phi(x, t)=\sum_{k=1}^{\infty}\left(A_{k} \cos (k \pi t)+B_{k} \sin (k \pi t)\right) \sqrt{2} \sin (k \pi x)
$$

and their energy (that is constant with time) is

$$
E_{\phi}(0)=\frac{\pi^{2}}{2} \sum_{k=1}^{\infty} k^{2}\left(A_{k}{ }^{2}+B_{k}{ }^{2}\right) .
$$

First let us observe that we can assume that $T<1$ : indeed if $T \geq 1$

$$
\begin{aligned}
\int_{0}^{1} \int_{\Omega} \phi_{t}(x, t)^{2} \mathrm{~d} x \mathrm{~d} t & =\int_{0}^{1} \int_{\Omega}\left(\sum_{k=1}^{\infty}\left(-k \pi A_{k} \sin (k \pi t)+k \pi B_{k} \cos (k \pi t)\right) \sqrt{2} \sin (k \pi x)\right)^{2} \mathrm{~d} x \mathrm{~d} t \\
& =\int_{0}^{1} \sum_{k=1}^{\infty} k^{2} \pi^{2}\left(-A_{k} \sin (k \pi t)+B_{k} \cos (k \pi t)\right)^{2} \mathrm{~d} t \\
& =\frac{\pi^{2}}{2} \sum_{k=1}^{\infty} k^{2}\left({A_{k}}^{2}+B_{k}{ }^{2}\right)=E_{\phi}(0) .
\end{aligned}
$$


Now assume that $T<1$ and compute the right-hand side of (10.5) with $T^{*}=T$ :

$$
\begin{aligned}
\int_{0}^{T} \int_{\Omega} a(t) \phi_{t}(x, t)^{2} \mathrm{~d} x \mathrm{~d} t & =\int_{0}^{T} \int_{\Omega}\left(\sum_{k=1}^{\infty}\left(-k \pi A_{k} \sin (k \pi t)+k \pi B_{k} \cos (k \pi t)\right) \sqrt{2} \sin (k \pi x)\right)^{2} \mathrm{~d} x \mathrm{~d} t \\
& =\int_{0}^{T} \sum_{k=1}^{\infty} k^{2} \pi^{2}\left(-A_{k} \sin (k \pi t)+B_{k} \cos (k \pi t)\right)^{2} \mathrm{~d} t \\
& =\pi^{2} \sum_{k=1}^{\infty} k^{2}\left(\alpha_{k} A_{k}{ }^{2}+\beta_{k} B_{k}{ }^{2}+2 \gamma_{k} A_{k} B_{k}\right),
\end{aligned}
$$

with

$$
\begin{aligned}
\alpha_{k} & :=\frac{T}{2}-\frac{1}{4 k \pi}[\sin (2 k \pi t)]_{0}^{T}, \\
\beta_{k} & :=\frac{T}{2}+\frac{1}{4 k \pi}[\sin (2 k \pi t)]_{0}^{T}, \\
\gamma_{k} & :=\frac{1}{4 k \pi}[\cos (2 k \pi t)]_{0}^{T} .
\end{aligned}
$$

Let us denote the symmetric matrix

$$
M_{k}=\left(\begin{array}{cc}
\alpha_{k} & \gamma_{k} \\
\gamma_{k} & \beta_{k}
\end{array}\right)=: \frac{T}{2} I d+N_{k}
$$

The matrix $M_{k}$ has two real eigenvalues $r_{k} \leq s_{k}$. The proof of (10.5) will be complete if we prove that there exists $\alpha>0$ such that $r_{k} \geq \alpha>0$ for all $k \in \mathbb{N}^{*}$. Indeed, if we denote $\psi_{k}:=\left(A_{k}, B_{k}\right) \in \mathbb{R}^{2}$, and $(\cdot \mid \cdot)$ the euclidean product of $\mathbb{R}^{2}$, we have

$$
\int_{0}^{T} \int_{\Omega} a(t) \phi_{t}(x, t)^{2} \mathrm{~d} x \mathrm{~d} t=\pi^{2} \sum_{k=1}^{\infty} k^{2}\left(M_{k} \cdot \psi_{k} \mid \psi_{k}\right) \geq \alpha \pi^{2} \sum_{k=1}^{\infty} k^{2}\left(A_{k}{ }^{2}+B_{k}{ }^{2}\right)=2 \alpha E_{\phi}(0) .
$$

So let us study the eigenvalues of $N_{k}$; the trace of $N_{k}$ is equal to 0 , thus $N_{k}$ has two opposite eigenvalues. We compute its determinant to know them:

$$
\operatorname{det} N_{k}=\frac{-1}{16 k^{2} \pi^{2}}\left[\left([\sin (2 k \pi t)]_{0}^{T}\right)^{2}+\left([\cos (2 k \pi t)]_{0}^{T}\right)^{2}\right]=-\left(\frac{\sin (k \pi T)}{2 k \pi}\right)^{2} .
$$

Hence

$$
r_{k}=\frac{T}{2}-\left|\frac{\sin (k \pi T)}{2 k \pi}\right|=\frac{T}{2}\left(1-\left|\frac{\sin (k \pi T)}{k \pi T}\right|\right) \geq \frac{T}{2}\left(1-C_{T}\right)
$$

where

$$
C_{T}:=\sup _{k}\left|\frac{\sin (k \pi T)}{k \pi T}\right|=\max _{k}\left|\frac{\sin (k \pi T)}{k \pi T}\right|<1 .
$$

10.3. Proof of Theorem 2.3 when $q=2$ and $\omega=(1 / 2-\lambda, 1 / 2+\lambda)$ in the case $1 / T \notin 2 \mathbb{N}$ Write

$$
1=2 \ell T+r T, \text { with } 0<r<2 .
$$


Then we have the following:

Lemma 10.1. When $0<r \leq 1$, let $N_{r}$ the smallest integer such that $r N_{r} \geq 1$, and when $1 \leq r<2$, let $N_{r}$ the smallest integer such that $(2-r) N_{r} \geq 1$. Then for all $t \in[0,1], a(t)+a(t+1)+\cdots+a\left(t+N_{r}\right) \geq 1$.

Proof of Lemma 10.1. We let it to the reader since it is very similar to Lemma 6.1: denote $\mathcal{G}_{2}:=\cup_{n}[2 n T, 2 n T+$ $T$ ) and $\mathcal{B}_{2}:=\mathbb{R}_{+} \backslash \mathcal{G}$, and verify that, given $t \in[0,1]$, it is not possible for $t, t+1, \cdots, t+N_{r}$ to be all in $\mathcal{B}_{2}$.

Now we are ready to prove that $(10.3)$ is satisfied with $T^{*}:=N_{r}+1$.

First verify that $\phi_{t}(x, t+2)=\phi_{t}(x, t)$, and $\phi_{t}(x, t+1)=-\phi_{t}(1-x, t)$. Then using the fact that $\omega$ is symmetrical with respect to $1 / 2$, we obtain that

$$
\int_{\omega} \phi_{t}(x, t+1)^{2} \mathrm{~d} x=\int_{\omega} \phi_{t}(x, t)^{2} \mathrm{~d} x
$$

Therefore we have

$$
\begin{aligned}
\int_{0}^{T^{*}} \int_{\omega} a(t) \phi_{t}(x, t)^{2} \mathrm{~d} x \mathrm{~d} t & =\sum_{m=0}^{N_{r}} \int_{m T}^{(m+1) T} a(t) \int_{\omega} \phi_{t}(x, t)^{2} \mathrm{~d} x \mathrm{~d} t \\
& =\sum_{m=0}^{N_{r}} \int_{0}^{1} a(t+m) \int_{\omega} \phi_{t}(x, t+m)^{2} \mathrm{~d} x \mathrm{~d} t \\
& =\sum_{m=0}^{N_{r}} \int_{0}^{1} a(t+m) \int_{\omega} \phi_{t}(x, t)^{2} \mathrm{~d} x \mathrm{~d} t \\
& =\int_{0}^{1}\left(\sum_{m=0}^{N_{r}} a(t+m)\right) \int_{\omega} \phi_{t}(x, t)^{2} \mathrm{~d} x \mathrm{~d} t \geq \int_{0}^{1} \int_{\omega} \phi_{t}(x, t)^{2} \mathrm{~d} x \mathrm{~d} t
\end{aligned}
$$

But from 5.1, we have

$$
\int_{0}^{1} \int_{\omega} \phi_{t}(x, t)^{2} \mathrm{~d} x \mathrm{~d} t \geq C_{\lambda} E_{\phi}(0)
$$

Once again there is also a simple proof based on spectral decomposition:

$$
\begin{aligned}
\int_{0}^{1} \int_{\omega} \phi_{t}(x, t)^{2} \mathrm{~d} x \mathrm{~d} t & =\int_{0}^{1} \int_{\omega} \sum_{k=1}^{\infty} \sum_{l=1}^{\infty}\left(-k \pi A_{k} \sin (k \pi t)+k \pi B_{k} \cos (k \pi t)\right) \\
& =\sum_{k=1}^{\infty} \sum_{l=1}^{\infty}\left(\int_{0}^{1}\left(-k \pi A_{l} \sin (l \pi t)+l \pi B_{l} \cos (l \pi t)\right)(2 \sin (k \pi x) \sin (l \pi x)) \mathrm{d} x \mathrm{~d} t\right. \\
& \left(\int_{\omega} 2 \sin (k \pi x) \sin (l \pi x) \mathrm{d} x\right)
\end{aligned}
$$

It is easy to verify first that when $k=l$ :

$$
\int_{0}^{1}\left(-k \pi A_{k} \sin (k \pi t)+k \pi B_{k} \cos (k \pi t)\right)^{2} \mathrm{~d} t=\frac{\pi^{2}}{2} k^{2}\left(A_{k}^{2}+B_{k}^{2}\right)
$$


and

$$
\int_{\omega} 2 \sin (k \pi x)^{2} \mathrm{~d} x=2 \lambda\left(1-(-1)^{k} \frac{\sin (2 k \pi \lambda)}{2 k \pi \lambda}\right) \geq 2 \lambda\left(1-C_{\lambda}\right)
$$

(remember that $C_{\lambda}<1$ ); next when $k \neq l$, we verify that

$$
\int_{0}^{1}\left(-k \pi A_{k} \sin (k \pi t)+k \pi B_{k} \cos (k \pi t)\right)\left(-l \pi A_{l} \sin (l \pi t)+l \pi B_{l} \cos (l \pi t)\right) \mathrm{d} t=0
$$

if $k+l$ is even, and

$$
\int_{\omega} 2 \sin (k \pi x) \sin (l \pi x) \mathrm{d} x=0
$$

if $k+l$ is odd. Hence

$$
\int_{0}^{1} \int_{\omega} \phi_{t}(x, t)^{2} \mathrm{~d} x \mathrm{~d} t=\lambda \pi^{2} \sum_{k=1}^{\infty} k^{2}\left(A_{k}{ }^{2}+B_{k}{ }^{2}\right)\left(1-(-1)^{k} \frac{\sin (2 k \pi \lambda)}{2 k \pi \lambda}\right) \geq \lambda \pi^{2}\left(1-C_{\lambda}\right) \sum_{k=1}^{\infty} k^{2}\left(A_{k}{ }^{2}+B_{k}{ }^{2}\right) .
$$

10.4. Proof of Theorem 2.3 when $q=2$ and $\omega=(1 / 2-\lambda, 1 / 2+\lambda)$ in the case $1 / T \in 2 \mathbb{N}$ and $2 \lambda<T$

Now we assume that

$$
1=2 \ell T \text { and } 2 \lambda<T .
$$

We will construct some solutions $\phi$ of $(2.6)$ such that $E_{\phi}(0) \neq 0$ and such that

$$
\forall T^{*}>0, \quad \int_{0}^{T^{*}} a(t) \int_{\omega} \phi_{t}^{2}(x, t) \mathrm{d} x \mathrm{~d} t=0 .
$$

It will first prove that Theorem 5.2 is optimal. Moreover it implies that $a \chi_{\omega} \phi_{t} \equiv 0$. Thus $\phi$ is also solution of (1.2) with $E_{\phi}(t)=E_{\phi}(0)>0$ for all $t \geq 0$, which proves Theorem 2.3 when $q=2$ in this case.

First remark that, since $1=2 \ell T, a$ is 1-periodic, thus to obtain (10.8), it is sufficient to prove

$$
\int_{0}^{1} a(t) \int_{\omega} \phi_{t}^{2}(x, t) \mathrm{d} x \mathrm{~d} t=0 .
$$

Let us consider $f:(-1,+\infty) \rightarrow \mathbb{R}$ an absolutely continuous function (that we will choose later) such that $f^{\prime} \in L_{\mathrm{loc}}^{2}(-1, \infty)$ and such that $f$ is 2 -periodic. Then we define

$$
\phi(x, t)=f(t+x)-f(t-x), \quad(x, t) \in(0,1) \times \mathbb{R}_{+} .
$$

We easily verify that $\phi$ is solution of (2.6). Note also that

$$
E_{\phi}(0)=\int_{-1}^{1} f^{\prime}(s)^{2} \mathrm{~d} s=\int_{-1 / 2-\lambda}^{3 / 2+\lambda} f^{\prime}(s)^{2} \mathrm{~d} s,
$$


and

$$
\int_{0}^{1} a(t) \int_{\omega} \phi_{t}^{2}(x, t) \mathrm{d} x \mathrm{~d} t=\sum_{m=0}^{\ell-1} \int_{2 m T}^{(2 m+1) T} a(t) \int_{1 / 2-\lambda}^{1 / 2+\lambda}\left(f^{\prime}(t+x)-f^{\prime}(t-x)\right)^{2} \mathrm{~d} x \mathrm{~d} t .
$$

For all $m \in\{0, \ldots, \ell-1\}$, we have

$$
\begin{gathered}
\left\{\begin{array}{l}
t \in(2 m T,(2 m+1) T) \\
x \in(1 / 2-\lambda, 1 / 2+\lambda)
\end{array}\right. \\
\Longrightarrow\left\{\begin{array}{l}
t+x \in\left(a_{m}, b_{m}\right):=(2 m T+1 / 2-\lambda,(2 m+1) T+1 / 2+\lambda) \\
t-x \in\left(\alpha_{m}, \beta_{m}\right):=(2 m T-1 / 2-\lambda,(2 m+1) T-1 / 2+\lambda)
\end{array}\right.
\end{gathered}
$$

Using that $2 \lambda<T$, we verify that

$$
-1 / 2-\lambda=\alpha_{0}<\beta_{0}<\alpha_{1}<\ldots<\alpha_{l-1}<\beta_{l-1}<a_{0}<b_{0}<a_{1}<\ldots<b_{l-1}<3 / 2-\lambda .
$$

Then we denote

$$
A=\left(\bigcup_{m=0}^{l-1}\left(a_{m}, b_{m}\right)\right) \cup\left(\bigcup_{m=0}^{l-1}\left(\alpha_{m}, \beta_{m}\right)\right)
$$

Now we choose $f$. It is sufficient to determine $f(s)$ for $s \in(-1 / 2-\lambda, 3 / 2-\lambda)$ since $f$ is 2 -periodic. First we decide to choose $f(s)=0$ for all $s \in A$ so that, from (10.11) and (10.12), we have (10.9). Then we can choose $f$ on $(-1 / 2-\lambda, 3 / 2-\lambda) \backslash A$ such that $f(-1 / 2-\lambda)=f(3 / 2-\lambda)$ and $E_{\phi}(0) \neq 0$. It is possible from (10.10) and from (10.13) since it implies that $(-1 / 2-\lambda, 3 / 2-\lambda) \backslash A \neq \emptyset$.

\subsection{Proof of Theorem 2.3 when $q=2$ and $\omega=(1 / 2-\lambda, 1 / 2+\lambda)$ and $1 / T \in 2 \mathbb{N}$ and $2 \lambda>T$}

We assume that

$$
1=2 \ell T \text { and } 2 \lambda>T \text {. }
$$

Then Theorem 2.3 when $q=2$ follows directly from Proposition 10.1 and Theorem 5.2.

\section{Proof of Theorem 5.2 (observability inequalities)}

\section{Notations and preliminaries}

We will first consider smooth solution $\phi$ of (2.6) and next we will deduce the result for all solution $\phi$ of (2.6) with $\left(\phi_{0}, \phi_{1}\right) \in H_{0}^{1}(0,1) \times L^{2}(0,1)$ by an argument of density.

Assume $T=1 /(2 \ell)$ and $2 \lambda>T$. There exists $\lambda^{\prime}$ such that $\lambda>\lambda^{\prime}>T / 2$. We introduce $\delta=\lambda^{\prime}-T / 2>0$ and $\eta=\delta / 8$.

We can apply Theorem 5.1 to the interval $\left(a^{\prime}, b^{\prime}\right)=\left(1 / 2-\lambda^{\prime}, 1 / 2+\lambda^{\prime}\right)$ in space and $(\eta, 1-T-\eta)$ in time, since we have

$$
1-T-2 \eta>2 \max \left(a^{\prime}, 1-b^{\prime}\right)=1-2 \lambda^{\prime} .
$$

Hence there exists $C>0$ such that, for all solution of (2.6),

$$
E_{\phi}(0) \leq C \int_{\eta}^{1-T-\eta} \int_{1 / 2-\lambda^{\prime}}^{1 / 2+\lambda^{\prime}} \phi_{t}^{2}(x, t) \mathrm{d} x \mathrm{~d} t
$$


For all smooth solution $\phi$ of (2.6), we also define

$$
\forall(x, t) \in[0,1] \times \mathbb{R}_{+}, \quad f(x, t)=\phi_{t}^{2}(x, t)+\phi_{x}^{2}(x, t) .
$$

So the starting point of the proof will be the following inequality: there exists $C>0$ such that, for all smooth solution $\phi$ of $(2.6)$,

$$
E_{\phi}(0) \leq C \int_{\eta}^{1-T-\eta} \int_{1 / 2-\lambda^{\prime}}^{1 / 2+\lambda^{\prime}} f(x, t) \mathrm{d} x \mathrm{~d} t
$$

Step 1. The main step of the proof is the following lemma (proved in Sect. 11.1):

Lemma 11.1. Using the previous assumptions and notations, there exists $C>0$ such that, for all smooth solution $\phi$ of $(2.6)$,

$$
\sum_{p=0}^{l-2} \int_{(2 p+1) T-\eta}^{(2 p+2) T+\eta} \int_{1 / 2-\lambda^{\prime}}^{1 / 2+\lambda^{\prime}} f(x, t) \mathrm{d} x \mathrm{~d} t \leq C \sum_{p=0}^{l-1} \int_{2 p T+\eta}^{(2 p+1) T-\eta} \int_{1 / 2-\lambda^{\prime}}^{1 / 2+\lambda^{\prime}} f(x, t) \mathrm{d} x \mathrm{~d} t
$$

If we combine (11.1) with Lemma 11.1, we obtain: there exists $C>0$ such that, for all $\phi$ solution of (2.6),

$$
\begin{aligned}
E_{\phi}(0) \leq & C \int_{\eta}^{1-T-\eta} \int_{1 / 2-\lambda^{\prime}}^{1 / 2+\lambda^{\prime}} f(x, t) \mathrm{d} x \mathrm{~d} t=C \sum_{p=0}^{l-1} \int_{2 p T+\eta}^{(2 p+1) T-\eta} \int_{1 / 2-\lambda^{\prime}}^{1 / 2+\lambda^{\prime}} f(x, t) \mathrm{d} x \mathrm{~d} t \\
& +C \sum_{p=0}^{l-2} \int_{(2 p+1) T-\eta}^{(2 p+2) T+\eta} \int_{1 / 2-\lambda^{\prime}}^{1 / 2+\lambda^{\prime}} f(x, t) \mathrm{d} x \mathrm{~d} t \\
\leq & C^{\prime} \sum_{p=0}^{l-1} \int_{2 p T+\eta}^{(2 p+1) T-\eta} \int_{1 / 2-\lambda^{\prime}}^{1 / 2+\lambda^{\prime}} \phi_{t}^{2}(x, t)+\phi_{x}^{2}(x, t) \mathrm{d} x \mathrm{~d} t .
\end{aligned}
$$

Step 2. We use the following lemma (proved in Sect. 11.2):

Lemma 11.2. For all $\nu>0, \varepsilon>0$ and for all $\nu \leq T_{1} \leq T_{2}, \nu \leq \alpha \leq \beta \leq 1-\nu$, there exists $C_{\varepsilon}>0$ such that, for all smooth solution $\phi$ of (2.6),

$$
\int_{T_{1}}^{T_{2}} \int_{\alpha}^{\beta} \phi_{x}^{2}(x, t) \mathrm{d} x \mathrm{~d} t \leq C_{\varepsilon} \int_{T_{1}-\nu}^{T_{2}+\nu} \int_{\alpha-\nu}^{\beta+\nu} \phi_{t}^{2}(x, t)+\phi^{2}(x, t) \mathrm{d} x \mathrm{~d} t+\varepsilon E_{\phi}(0) .
$$

For all $p \in\{0, \ldots, l-1\}$, we apply Lemma 11.2 to $T_{1}=2 p T+\eta, T_{2}=(2 p+1) T-\eta, \alpha=a^{\prime}=1 / 2-\lambda^{\prime}$ and $\beta=b^{\prime}=1 / 2+\lambda^{\prime}$ and with $2 \nu<\min \left(\eta, \lambda-\lambda^{\prime}\right)$. Combined with (11.3), this gives: for all $\varepsilon>0$, there exists $C_{\varepsilon}>0$ such that, for all $\phi$ solution of $(2.6)$,

$$
\begin{aligned}
E_{\phi}(0) & \leq C_{\varepsilon} \sum_{p=0}^{l-1} \int_{2 p T+\eta-\nu}^{(2 p+1) T-\eta+\nu} \int_{1 / 2-\lambda^{\prime}-\nu}^{1 / 2+\lambda^{\prime}+\nu} \phi_{t}^{2}(x, t)+\phi^{2}(x, t) \mathrm{d} x \mathrm{~d} t+\varepsilon E_{\phi}(0) \\
& \leq C_{\varepsilon} \sum_{p=0}^{l-1} \int_{2 p T+\eta-\nu}^{(2 p+1) T-\eta+\nu} \int_{1 / 2-\lambda+\nu}^{1 / 2+\lambda-\nu} \phi_{t}^{2}(x, t)+\phi^{2}(x, t) \mathrm{d} x \mathrm{~d} t+\varepsilon E_{\phi}(0) .
\end{aligned}
$$


If we chose $\varepsilon$ small enough, we deduce: there exists $C>0$ such that, for all smooth solution $\phi$ of $(2.6)$,

$$
E_{\phi}(0) \leq C \sum_{p=0}^{l-1} \int_{2 p T+\eta-\nu}^{(2 p+1) T-\eta+\nu} \int_{1 / 2-\lambda+\nu}^{1 / 2+\lambda-\nu} \phi_{t}^{2}(x, t)+\phi^{2}(x, t) \mathrm{d} x \mathrm{~d} t .
$$

Moreover by density, this inequality still holds for all solution $\phi$ of $(2.6)$ with $\left(\phi_{0}, \phi_{1}\right) \in H_{0}^{1}(0,1) \times L^{2}(0,1)$.

Step 3. Now we use the following lemma (proved in Sect. 11.3):

Lemma 11.3. Let $I^{\prime} \varsubsetneqq I \subset\left[0, T^{\prime}\right]$ with $T^{\prime}>0$ and let $\omega^{\prime} \subsetneq \omega \subset(0,1)$. Assume that there exists $C>0$ such that, for all $\phi$ solution of $(2.6)$ with $\left(\phi_{0}, \phi_{1}\right) \in H_{0}^{1}(0,1) \times L^{\frac{F}{2}}(0,1)$,

$$
E_{\phi}(0) \leq C \int_{I^{\prime}} \int_{\omega^{\prime}} \phi_{t}^{2}(x, t)+\phi^{2}(x, t) \mathrm{d} x \mathrm{~d} t .
$$

Then there exists $C>0$ such that, for all $\phi$ solution of $(2.6)$ with $\left(\phi_{0}, \phi_{1}\right) \in H_{0}^{1}(0,1) \times L^{2}(0,1)$,

$$
\int_{I^{\prime}} \int_{\omega^{\prime}} \phi^{2}(x, t) \mathrm{d} x \mathrm{~d} t \leq C \int_{I} \int_{\omega} \phi_{t}^{2}(x, t) \mathrm{d} x \mathrm{~d} t .
$$

If we apply this lemma to $\omega^{\prime}=(1 / 2-\lambda+\nu, 1 / 2+\lambda-\nu), \omega=(1 / 2-\lambda, 1 / 2+\lambda), I^{\prime}=\cup_{p=0}^{l-1}[2 p T+\eta-\nu$, $(2 p+1) T-\eta+\nu]$ and $I=\cup_{p=0}^{l-1}[2 p T,(2 p+1) T]$, equation (5.13) follows directly from (11.5), which ends the proof of Theorem 5.2.

\subsection{Proof of Lemma $\mathbf{1 1 . 1}$}

The proof is divided in two steps.

The first step is based on a method introduced by Haraux in [9] that consists of exchanging the role of the two variables $x$ and $t$. In [9], Haraux used this method to an estimate of an integral over $(0,1) \times(T / 2-\nu, T / 2+\nu)$ (for some $\nu$ small) by an integral over $(a, b) \times(0, T)$. Here we use it to obtain an estimate of an integral over $(1 / 2-\delta / 2,1 / 2+\delta / 2) \times(0, T)$ by an integral over $(1 / 2-\lambda, 1 / 2+\lambda) \times I$. The proof is based on Lemma 11.4.

In the second step, we estimate the integral over $(1 / 2-\lambda, 1 / 2+\lambda) \times(0, T)$ by an integral over $(1 / 2-\delta / 2,1 / 2+$ $\delta / 2) \times(0, T)$. In the case of [9], we had directly an estimate of the integral over $(0,1) \times(0, T)$ by an integral over $(0,1) \times(T / 2-\nu, T / 2+\nu)$ that followed from the energy equality obtained when we multiply the equation by $\phi_{t}$ and take the integral over $x \in(0,1)$. Here the role of $x$ and $t$ are opposite. So the proof is based on Lemma 11.5, that gives the equality that we obtain when we multiply the equation by $\phi_{x}$ and take the integral over $t$.

Step 1. In a first step, we will prove the following inequalities: there exists $C>$ such that, for all solution $\phi$ of $(2.6)$,

$\forall p \in\{1, \ldots, l-2\}, \quad \forall t \in[(2 p+1) T-\eta,(2 p+1) T+T / 2]$,

$$
\int_{1 / 2-\delta / 2}^{1 / 2+\delta / 2} f(x, t) \mathrm{d} x \leq C \int_{(2 p+1) T-3 \eta}^{(2 p+1) T-\eta} \int_{1 / 2-\lambda^{\prime}+\eta}^{1 / 2+\lambda^{\prime}-\eta} f(x, \sigma) \mathrm{d} x \mathrm{~d} \sigma .
$$

$\forall p \in\{1, \ldots, l-2\}, \quad \forall t \in[(2 p+1) T+T / 2,(2 p+1) T+\eta]$

$$
\int_{1 / 2-\delta / 2}^{1 / 2+\delta / 2} f(x, t) \mathrm{d} x \leq C \int_{(2 p+2) T+\eta}^{(2 p+2) T+3 \eta} \int_{1 / 2-\lambda^{\prime}+\eta}^{1 / 2+\lambda^{\prime}-\eta} f(x, \sigma) \mathrm{d} x d \sigma .
$$

Proof of (11.8). The proof is based on the following lemma (proved in Sect. 11.4): 
Lemma 11.4. For all $t, \xi$ such that $0 \leq a^{\prime}+(t-\xi) \leq b^{\prime}-(t-\xi) \leq 1$,

$$
\frac{\mathrm{d}}{\mathrm{d} t} \int_{a^{\prime}+(t-\xi)}^{b^{\prime}-(t-\xi)} f(x, t) \mathrm{d} x \leq 0 .
$$

We can apply this lemma for all $t, \xi$ such that $0 \leq t-\xi \leq\left(b^{\prime}-a^{\prime}\right) / 2=\lambda^{\prime}$, and take the integral between $t_{0}$ and $t$ such that $t_{0} \leq t$, which gives:

$\forall t_{0}, t, \xi$ such that $0 \leq t_{0}-\xi \leq t-\xi \leq \lambda^{\prime}$,

$$
\int_{1 / 2-\lambda^{\prime}+(t-\xi)}^{1 / 2+\lambda^{\prime}-(t-\xi)} f(x, t) \mathrm{d} x \leq \int_{1 / 2-\lambda^{\prime}+\left(t_{0}-\xi\right)}^{1 / 2+\lambda^{\prime}-\left(t_{0}-\xi\right)} f\left(x, t_{0}\right) \mathrm{d} x .
$$

For all $p \in\{1, \ldots, l-2\}$, we choose

$$
t \in[(2 p+1) T-\eta,(2 p+1) T+T / 2], \quad \text { and } \quad \xi \in[(2 p+1) T-4 \eta,(2 p+1) T-2 \eta],
$$

and we take $t_{0}=\xi+\eta$. Note that $t_{0} \in[(2 p+1) T-3 \eta,(2 p+1) T-\eta]$.

Under condition (11.11), we can verify, using the definition of $\eta$,

$$
0 \leq \eta=t_{0}-\xi \leq t-\xi \leq \frac{T}{2}+4 \eta=\lambda^{\prime}-\frac{\delta}{2} \leq \lambda^{\prime}
$$

Therefore we can apply (11.10), which gives: for all $t, \xi$ satisfying (11.11),

$$
\int_{1 / 2-\lambda^{\prime}+(t-\xi)}^{1 / 2+\lambda^{\prime}-(t-\xi)} f(x, t) \mathrm{d} x \leq \int_{1 / 2-\lambda^{\prime}+\eta}^{1 / 2+\lambda^{\prime}-\eta} f(x, \xi+\eta) \mathrm{d} x .
$$

From (11.12), we deduce that, for all $t, \xi$ satisfying (11.11), we have

$$
\frac{1}{2}-\lambda^{\prime}+(t-\xi) \leq \frac{1}{2}-\frac{\delta}{2} \leq \frac{1}{2}+\frac{\delta}{2} \leq 1 / 2+\lambda^{\prime} / 2-(t-\xi)
$$

which implies

$$
\int_{1 / 2-\delta / 2}^{1 / 2+\delta / 2} f(x, t) \mathrm{d} x \leq \int_{1 / 2-\lambda^{\prime}+(t-\xi)}^{1 / 2+\lambda^{\prime}-(t-\xi)} f(x, t) \mathrm{d} x
$$

On the other hand, using the average formula, we have:

$\exists \xi_{0}$ such that $(2 p+1) T-3 \eta \leq \xi_{0}+\eta \leq(2 p+1) T-\eta$ and

$$
\int_{1 / 2-\lambda^{\prime}+\eta}^{1 / 2+\lambda^{\prime}-\eta} f\left(x, \xi_{0}+\eta\right) \mathrm{d} x=\frac{1}{2 \eta} \int_{(2 p+1) T-3 \eta}^{(2 p+1) T-\eta} \int_{1 / 2-\lambda^{\prime}+\eta}^{1 / 2+\lambda^{\prime}-\eta} f(x, \sigma) \mathrm{d} x d \sigma .
$$

Since $\xi_{0} \in[(2 p+1) T-4 \eta,(2 p+1) T-2 \eta]$, we can choose $\xi=\xi_{0}$ in (11.13), and using (11.14) and (11.15), we obtain (11.8).

Proof of (11.9). We can prove (11.9) by a similar proof proceeding backward in $t$. 
Step 2. We use the following lemma (proved in Sect. 11.5):

Lemma 11.5. For all $0 \leq T_{1} \leq T_{2}$ and for all $x_{0} \in\left(1 / 2-\lambda^{\prime}, 1 / 2+\lambda^{\prime}\right)$,

$$
\int_{T_{1}}^{T_{2}} f\left(x_{0}, t\right) \mathrm{d} t \leq \frac{1}{\delta} \int_{T_{1}}^{T_{2}} \int_{1 / 2-\delta / 2}^{1 / 2+\delta / 2} f(x, t) \mathrm{d} x \mathrm{~d} t+\int_{1 / 2-\lambda^{\prime}}^{1 / 2+\lambda^{\prime}} f\left(x, T_{1}\right)+f\left(x, T_{2}\right) \mathrm{d} x .
$$

For all $p \in\{1, \ldots, l-1\}$, we apply this lemma to $T_{1}=(2 p+1) T-\sigma_{1}$ and $T_{2}=(2 p+2) T+\sigma_{2}$ where we choose $\sigma_{1}$ and $\sigma_{2}$ as following: using the average formula, there exists $\sigma_{1}$ such that $2 p T+\eta \leq(2 p+1) T-\sigma_{1} \leq(2 p+1) T-\eta$ and

$$
\int_{1 / 2-\lambda^{\prime}}^{1 / 2+\lambda^{\prime}} f\left(x,(2 p+1) T-\sigma_{1}\right) \mathrm{d} x=\frac{1}{T-2 \eta} \int_{2 p T+\eta}^{(2 p+1) T-\eta} \int_{1 / 2-\lambda^{\prime}}^{1 / 2+\lambda^{\prime}} f(x, t) \mathrm{d} x \mathrm{~d} t,
$$

and there exists $\sigma_{2}$ such that $(2 p+2) T+\eta \leq(2 p+2) T+\sigma_{2} \leq(2 p+3) T-\eta$ and

$$
\int_{1 / 2-\lambda^{\prime}}^{1 / 2+\lambda^{\prime}} f\left(x,(2 p+2) T+\sigma_{2}\right) \mathrm{d} x=\frac{1}{T-2 \eta} \int_{(2 p+2) T+\eta}^{(2 p+3) T-\eta} \int_{1 / 2-\lambda^{\prime}}^{1 / 2+\lambda^{\prime}} f(x, t) \mathrm{d} x \mathrm{~d} t .
$$

Applying Lemma 11.5 and using that $\sigma_{1}, \sigma_{2} \geq \eta$, we deduce, for all $x_{0} \in\left(1 / 2-\lambda^{\prime}, 1 / 2+\lambda^{\prime}\right)$,

$$
\begin{aligned}
\int_{(2 p+1) T-\eta}^{(2 p+2) T+\eta} f\left(x_{0}, t\right) \mathrm{d} t \leq & \int_{(2 p+1) T-\sigma_{1}}^{(2 p+2) T+\sigma_{2}} f\left(x_{0}, t\right) \mathrm{d} t \leq \frac{1}{\delta} \int_{(2 p+1) T-\sigma_{1}}^{(2 p+2) T+\sigma_{2}} \int_{1 / 2-\delta / 2}^{1 / 2+\delta / 2} f(x, t) \mathrm{d} x \mathrm{~d} t \\
& +\int_{1 / 2-\lambda^{\prime}}^{1 / 2+\lambda^{\prime}} f\left(x,(2 p+1) T-\sigma_{1}\right)+f\left(x,(2 p+2) T+\sigma_{2}\right) \mathrm{d} x \\
\leq & \frac{1}{\delta} \int_{(2 p+1) T-\sigma_{1}}^{(2 p+1) T-\eta} \int_{1 / 2}^{1 / 2+\delta / 2} f(x, t) \mathrm{d} x \mathrm{~d} t+\frac{1}{\delta} \int_{(2 p+1) T-\eta}^{(2 p+2) T+\eta} \int_{1 / 2-\delta / 2}^{1 / 2+\delta / 2} f(x, t) \mathrm{d} x \mathrm{~d} t \\
& +\frac{1}{\delta} \int_{(2 p+2) T+\eta}^{(2 p+2) T+\sigma_{2}} \int_{1 / 2-\delta / 2}^{1 / 2+\delta / 2} f(x, t) \mathrm{d} x \mathrm{~d} t \\
& +\frac{1}{T-2 \eta} \int_{2 p T+\eta}^{(2 p+1) T-\eta} \int_{1 / 2-\lambda^{\prime}}^{1 / 2+\lambda^{\prime}} f(x, t) \mathrm{d} x \mathrm{~d} t+\frac{1}{T-2 \eta} \int_{(2 p+2) T+\eta}^{(2 p+3) T-\eta} \int_{1 / 2-\lambda^{\prime}}^{1 / 2+\lambda^{\prime}} f(x, t) \mathrm{d} x \mathrm{~d} t .
\end{aligned}
$$

We use that $(2 p+1) T-\sigma_{1} \geq 2 p T+\eta,(2 p+2) T+\sigma_{2} \leq(2 p+3) T-\eta$ and that $1 / 2-\lambda^{\prime} \leq 1 / 2-\delta / 2 \leq$ $1 / 2+\delta / 2 \leq 1 / 2+\lambda^{\prime}$, to obtain

$$
\int_{(2 p+1) T-\sigma_{1}}^{(2 p+1) T-\eta} \int_{1 / 2-\delta / 2}^{1 / 2+\delta / 2} f(x, t) \mathrm{d} x \mathrm{~d} t \leq \int_{2 p T+\eta}^{(2 p+1) T-\eta} \int_{1 / 2-\lambda^{\prime}}^{1 / 2+\lambda^{\prime}} f(x, t) \mathrm{d} x \mathrm{~d} t,
$$

and

$$
\int_{(2 p+2) T+\eta}^{(2 p+2) T+\sigma_{2}} \int_{1 / 2-\delta / 2}^{1 / 2+\delta / 2} f(x, t) \mathrm{d} x \mathrm{~d} t \leq \int_{(2 p+2) T+\eta}^{(2 p+3) T-\eta} \int_{1 / 2-\lambda^{\prime}}^{1 / 2+\lambda^{\prime}} f(x, t) \mathrm{d} x \mathrm{~d} t .
$$

And using (11.8) and (11.9), we obtain, for all $x_{0} \in\left(1 / 2-\lambda^{\prime}, 1 / 2+\lambda^{\prime}\right)$,

$$
\int_{(2 p+1) T-\eta}^{(2 p+2) T+\eta} f\left(x_{0}, t\right) \mathrm{d} t \leq C \int_{2 p T+\eta}^{(2 p+1) T-\eta} \int_{1 / 2-\lambda^{\prime}}^{1 / 2+\lambda^{\prime}} f(x, t) \mathrm{d} x \mathrm{~d} t+C \int_{(2 p+2) T+\eta}^{(2 p+3) T-\eta} \int_{1 / 2-\lambda^{\prime}}^{1 / 2+\lambda^{\prime}} f(x, t) \mathrm{d} x \mathrm{~d} t .
$$

Finally, taking the integral over $x_{0} \in\left(1 / 2-\lambda^{\prime}, 1 / 2+\lambda^{\prime}\right)$, we obtain (11.2). 


\subsection{Proof of Lemma $\mathbf{1 1 . 2}$}

We use a localization argument like in Haraux [9]. We multiply equation (2.6) by $\phi$ and we use the relations $\phi_{t t} \phi=\left(\phi \phi_{t}\right)_{t}-\phi_{t}^{2}$ and $\phi_{x x} \phi=\left(\phi \phi_{x}\right)_{x}-\phi_{x}^{2}$. We obtain

$$
\phi_{x}^{2}=\phi_{t}^{2}-\left(\phi \phi_{t}\right)_{t}+\left(\phi \phi_{x}\right)_{x} .
$$

Let $\zeta$ be a fixed $\mathcal{C}^{\infty}$ function compactly supported in $(\alpha-\nu, \beta+\nu) \times\left(T_{1}-\nu, T_{2}+\nu\right)$ such that $0 \leq \zeta \leq 1$ on $(\alpha-\nu, \beta+\nu) \times\left(T_{1}-\nu, T_{2}+\nu\right)$ and $\zeta \equiv 1$ on $(\alpha, \beta) \times\left(T_{1}, T_{2}\right)$. We multiply the previous equation by $\zeta$ and integrate it over $(x, t) \in(\alpha-\nu, \beta+\nu) \times\left(T_{1}-\nu, T_{2}+\nu\right)$, which gives

$$
\begin{aligned}
\int_{T_{1}}^{T_{2}} \int_{\alpha}^{\beta} \phi_{x}^{2} \mathrm{~d} x \mathrm{~d} t & =\int_{T_{1}}^{T_{2}} \int_{\alpha}^{\beta} \phi_{x}^{2} \zeta \mathrm{d} x \mathrm{~d} t \leq \int_{T_{1}-\nu}^{T_{2}+\nu} \int_{\alpha-\nu}^{\beta+\nu} \phi_{x}^{2} \zeta \mathrm{d} x \mathrm{~d} t \\
& \leq \int_{T_{1}-\nu}^{T_{2}+\nu} \int_{\alpha-\nu}^{\beta+\nu}\left(\phi_{t}^{2}-\left(\phi \phi_{t}\right)_{t}+\left(\phi \phi_{x}\right)_{x}\right) \zeta \mathrm{d} x \mathrm{~d} t \\
\leq & \int_{T_{1}-\nu}^{T_{2}+\nu} \int_{\alpha-\nu}^{\beta+\nu} \phi_{t}^{2} \mathrm{~d} x \mathrm{~d} t \\
& +\left|\int_{T_{1}-\nu}^{T_{2}+\nu} \int_{\alpha-\nu}^{\beta+\nu}\left(\phi \phi_{t}\right)_{t} \zeta \mathrm{d} x \mathrm{~d} t\right|+\left|\int_{T_{1}-\nu}^{T_{2}+\nu} \int_{\alpha-\nu}^{\beta+\nu}\left(\phi \phi_{x}\right)_{x} \zeta \mathrm{d} x \mathrm{~d} t\right|
\end{aligned}
$$

Moreover we have

$$
\begin{aligned}
\left|\int_{T_{1}-\nu}^{T_{2}+\nu} \int_{\alpha-\nu}^{\beta+\nu}\left(\phi \phi_{t}\right)_{t} \zeta \mathrm{d} x \mathrm{~d} t\right| & =\left|\int_{T_{1}-\nu}^{T_{2}+\nu} \int_{\alpha-\nu}^{\beta+\nu}\left(\phi \phi_{t}\right) \zeta_{t} \mathrm{~d} x \mathrm{~d} t\right| \\
& \leq \frac{1}{2}\left\|\zeta_{t}\right\|_{\infty} \int_{T_{1}-\nu}^{T_{2}+\nu} \int_{\alpha-\nu}^{\beta+\nu}\left(\phi^{2}+\phi_{t}^{2}\right) \mathrm{d} x \mathrm{~d} t
\end{aligned}
$$

And we also have, for all $\varepsilon>0$,

$$
\begin{aligned}
\left|\int_{T_{1}-\nu}^{T_{2}+\nu} \int_{\alpha-\nu}^{\beta+\nu}\left(\phi \phi_{x}\right)_{x} \zeta \mathrm{d} x \mathrm{~d} t\right| & =\left|\int_{T_{1}-\nu}^{T_{2}+\nu} \int_{\alpha-\nu}^{\beta+\nu}\left(\phi \phi_{x}\right) \zeta_{x} \mathrm{~d} x \mathrm{~d} t\right| \\
& \leq \frac{1}{4 \varepsilon}\left\|\zeta_{x}\right\|_{\infty}^{2} \int_{T_{1}-\nu}^{T_{2}+\nu} \int_{\alpha-\nu}^{\beta+\nu} \phi^{2} \mathrm{~d} x \mathrm{~d} t+\varepsilon \int_{T_{1}-\nu}^{T_{2}+\nu} \int_{\alpha-\nu}^{\beta+\nu} \phi_{x}^{2} \mathrm{~d} x \mathrm{~d} t .
\end{aligned}
$$

Then Lemma 11.2 follows from $(11.16,11.17)$ and $(11.18)$ with $\varepsilon$ chosen small enough.

\subsection{Proof of Lemma $\mathbf{1 1 . 3}$}

The proof is based on a compactness argument. We assume that (11.7) is false. Then for all $n \in \mathbb{N}$, there exists $\phi_{n}$ solution of (2.6), such that

$$
\begin{gathered}
\forall n \in \mathbb{N}, \quad \int_{I} \int_{\omega} \phi_{n}^{2}(x, t) \mathrm{d} x \mathrm{~d} t=1 \\
\text { and } \forall n \in \mathbb{N}, \quad \int_{I} \int_{\omega}\left(\phi_{n}\right)_{t}^{2}(x, t) \mathrm{d} x \mathrm{~d} t \leq \frac{1}{n} .
\end{gathered}
$$


We introduce, for all $\phi$ solution of (2.6),

$$
\|\phi\|_{X}^{2}=\int_{I} \int_{\omega} \phi_{t}^{2}(x, t)+\phi^{2}(x, t) \mathrm{d} x \mathrm{~d} t
$$

From (11.6), it defines a norm that is equivalent to the norm $E_{\phi}(0)$ on the closure $\mathrm{X}$ of the space of all solutions of (2.6). From (11.19) and (11.20), the sequence $\left(\phi_{n},\left(\phi_{n}\right)_{t}\right)_{n}$ is bounded in $X$. So, for a subsequence still denoted by $\left(\phi_{n},\left(\phi_{n}\right)_{t}\right)$, we can verify that

$$
\left(\phi_{n},\left(\phi_{n}\right)_{t}\right) \rightarrow\left(\phi, \phi_{t}\right) \text { weakly in } X \text { as } n \rightarrow+\infty,
$$

where $\phi$ is a solution of (2.6).

We will prove the following contradiction:

$$
\int_{I} \int_{\omega} \phi^{2}(x, t) \mathrm{d} x \mathrm{~d} t=1
$$

and

$$
\forall(x, t) \in(0,1) \times \mathbb{R}_{+}, \quad \phi(x, t) \equiv 0,
$$

which will prove Lemma 11.3.

First, from (11.6), there exists $C, C^{\prime}>0$ such that, for all $n \in \mathbb{N}$,

$$
\int_{0}^{1-T} \int_{0}^{1}\left(\phi_{n}\right)_{t}^{2}(x, t)+\left(\phi_{n}\right)_{x}^{2}(x, t) \mathrm{d} x \mathrm{~d} t=2(1-T) E_{\phi_{n}}(0) \leq C\left\|\phi_{n}\right\|_{X}^{2} \leq C^{\prime} .
$$

Therefore $\left(\phi_{n}\right)_{n}$ is bounded in $H^{1}((0,1) \times(0,1-T))$, and consequently precompact in $L^{2}((0,1) \times(0,1-T))$. We deduce that, for a subsequence still denoted by $\left(\phi_{n}\right)$, we have $\phi_{n} \rightarrow \phi$ strongly in $L^{2}((0,1) \times(0,1-T))$ as $n \rightarrow+\infty$. Then (11.21) follows from (11.19).

On the other hand, equation (11.20) implies that $\left(\phi_{n}\right)_{t} \rightarrow 0$ strongly in $L^{2}(\omega \times I)$ as $n \rightarrow+\infty$. Thus we have $\phi_{t} \equiv 0$ on $\omega \times I$ and $\phi$ is solution of $(2.6)$ with $\left(\phi(0), \phi_{t}(0)\right) \in H_{0}^{1}(1,0) \times L^{2}(0,1)$.

One can construct a sequence of smooth solutions of $\phi^{\epsilon}$ of $(2.6)$ with $\left(\phi^{\epsilon}(0), \phi_{t}^{\epsilon}(0)\right) \in H^{2}(0,1) \cap H_{0}^{1}(1,0) \times$ $H_{0}^{1}(1,0)$ such that

$$
\left(\phi^{\epsilon}, \phi_{t}^{\epsilon}\right) \rightarrow\left(\phi, \phi_{t}\right) \quad \text { as } \epsilon \rightarrow 0,
$$

and such that $\phi_{t}^{\epsilon} \equiv 0$ on $\omega_{\epsilon} \times I_{\epsilon}$ where $\omega^{\prime} \times I^{\prime} \subset \omega_{\epsilon} \times I_{\epsilon} \subset \omega \times I$ for all $\epsilon$ small enough.

We denote $\psi^{\epsilon}=\phi_{t}^{\epsilon}$. Then we have $\psi^{\epsilon} \equiv \psi_{t}^{\epsilon} 0$ on $I^{\prime} \times \omega^{\prime}$ and $\psi^{\epsilon}$ is solution of $(2.6)$ with $\left(\psi^{\epsilon}(0), \psi_{t}^{\epsilon}(0)\right) \in$ $H_{0}^{1}(1,0) \times L^{2}(0,1)$. Thus we can apply (11.6) to $\psi^{\epsilon}$, which gives:

$$
E_{\psi}^{\epsilon}(0) \leq C \int_{I} \int_{\omega}\left(\psi_{t}^{\epsilon}\right)^{2}(x, t)+\left(\psi^{\epsilon}\right)^{2}(x, t) \mathrm{d} x \mathrm{~d} t=0
$$

We deduce that $\phi_{t}^{\epsilon}=\psi^{\epsilon} \equiv 0$ on $(0,1) \times \mathbb{R}_{+}$. Therefore $\phi_{t} \equiv 0$ on $(0,1) \times \mathbb{R}_{+}$. And since $\phi$ is solution of $(2.6)$, it implies $(11.22)$, i.e. $\phi \equiv 0$ on $(0,1) \times \mathbb{R}_{+}$. (Indeed, we deduce $-\phi_{x x}(x, t)=0$ on $(0,1) \times \mathbb{R}_{+}$with $\phi(0, t)=\phi(1, t)=0$.) 


\subsection{Proof of Lemma $\mathbf{1 1 . 4}$}

For all $t, \xi$ such that $0 \leq a^{\prime}+(t-\xi) \leq b^{\prime}-(t-\xi) \leq 1$, we compute

$$
\begin{aligned}
\frac{\mathrm{d}}{\mathrm{d} t} \int_{a^{\prime}+(t-\xi)}^{b^{\prime}-(t-\xi)} f(x, t) \mathrm{d} x= & -f\left(b^{\prime}-(t-\xi), t\right)-f\left(a^{\prime}+(t-\xi), t\right)+\int_{a^{\prime}+(t-\xi)}^{b^{\prime}-(t-\xi)} \frac{\partial}{\partial t} f(x, t) \mathrm{d} x \\
= & -\left(\phi_{t}^{2}+\phi_{x}^{2}\right)\left(b^{\prime}-(t-\xi), t\right)-\left(\phi_{t}^{2}+\phi_{x}^{2}\right)\left(a^{\prime}+(t-\xi), t\right) \\
& +\int_{a^{\prime}+(t-\xi)}^{b^{\prime}-(t-\xi)}\left(2 \phi_{t} \phi_{t t}+2 \phi_{x} \phi_{x t}\right)(x, t) \mathrm{d} x, \\
= & -\left(\phi_{t}^{2}+\phi_{x}^{2}\right)\left(b^{\prime}-(t-\xi), t\right)-\left(\phi_{t}^{2}+\phi_{x}^{2}\right)\left(a^{\prime}+(t-\xi), t\right) \\
& +2\left(\phi_{t} \phi_{x}\right)\left(b^{\prime}-(t-\xi), t\right)-2\left(\phi_{t} \phi_{x}\right)\left(a^{\prime}+(t-\xi), t\right) \\
& +2 \int_{a^{\prime}+(t-\xi)}^{b^{\prime}-(t-\xi)}\left(\phi_{t}\left(\phi_{t t}-\phi_{x x}\right)\right)(x, t) \mathrm{d} x \\
= & -\left(\phi_{t}\left(b^{\prime}-(t-\xi), t\right)-\phi_{x}\left(b^{\prime}-(t-\xi), t\right)\right)^{2} \\
& -\left(\phi_{t}\left(a^{\prime}+(t-\xi), t\right)+\phi_{x}\left(a^{\prime}+(t-\xi), t\right)\right)^{2} \leq 0 .
\end{aligned}
$$

\subsection{Proof of Lemma $\mathbf{1 1 . 5}$}

Multiplying equation (2.6) by $\phi_{x}$ and integrating over $t \in\left[T_{1}, T_{2}\right]$, we obtain, for all $x \in(0,1)$,

$$
\frac{1}{2} \frac{\mathrm{d}}{\mathrm{d} x} \int_{T_{1}}^{T_{2}}\left(\phi_{t}^{2}+\phi_{x}^{2}\right) \mathrm{d} t=\left[\phi_{t} \phi_{x}\right]_{t=T_{1}}^{t=T_{2}}
$$

Thus, for all $x_{0}, x \in(0,1)$,

$$
\int_{T_{1}}^{T_{2}} f\left(x_{0}, t\right) \mathrm{d} t=\int_{T_{1}}^{T_{2}} f(x, t) \mathrm{d} t+2 \int_{y=x}^{y=x_{0}}\left(\phi_{t} \phi_{x}\right)\left(y, T_{2}\right)-\left(\phi_{t} \phi_{x}\right)\left(y, T_{1}\right) \mathrm{d} y .
$$

For all $x_{0} \in\left(1 / 2-\lambda^{\prime}, 1 / 2+\lambda^{\prime}\right)$, the integral over $x \in(1 / 2-\delta / 2,1 / 2+\delta / 2)$ gives

$$
\delta \int_{T_{1}}^{T_{2}} f\left(x_{0}, t\right) \mathrm{d} t=\int_{T_{1}}^{T_{2}} \int_{1 / 2-\delta / 2}^{1 / 2+\delta / 2} f(x, t) \mathrm{d} x \mathrm{~d} t+2 \int_{x=1 / 2-\delta / 2}^{x=1 / 2+\delta / 2} \int_{y=x}^{y=x_{0}}\left(\left(\phi_{t} \phi_{x}\right)\left(y, T_{2}\right)-\left(\phi_{t} \phi_{x}\right)\left(y, T_{1}\right)\right) \mathrm{d} y \mathrm{~d} x
$$

Using that $2\left|\phi_{t} \phi_{x}\right| \leq f$ and that $\left(x_{0}, x\right) \subset\left(1 / 2-\lambda^{\prime}, 1 / 2+\lambda^{\prime}\right)$ for all $x_{0} \in\left(1 / 2-\lambda^{\prime}, 1 / 2+\lambda^{\prime}\right)$ and all $x \in(1 / 2-\delta / 2,1 / 2+\delta / 2)$, we deduce:

$$
\begin{aligned}
\delta \int_{T_{1}}^{T_{2}} f\left(x_{0}, t\right) \mathrm{d} t & \leq \int_{T_{1}}^{T_{2}} \int_{1 / 2-\delta / 2}^{1 / 2+\delta / 2} f(x, t) \mathrm{d} x \mathrm{~d} t+\int_{x=1 / 2-\delta / 2}^{x=1 / 2+\delta / 2}\left|\int_{y=x}^{y=x_{0}} f\left(y, T_{2}\right)+f\left(y, T_{1}\right) \mathrm{d} y\right| \mathrm{d} x \\
& \leq \int_{T_{1}}^{T_{2}} \int_{1 / 2-\delta / 2}^{1 / 2+\delta / 2} f(x, t) \mathrm{d} x \mathrm{~d} t+\delta \int_{1 / 2-\lambda^{\prime}}^{1 / 2+\lambda^{\prime}} f\left(y, T_{2}\right)+f\left(y, T_{1}\right) \mathrm{d} y .
\end{aligned}
$$

12. TOOLS FOR The ProOf OF TheOrem 2.3 AND TheOrem 2.4

When the feedback is uniformly distributed in the domain $(\lambda=1 / 2)$, the proof is the same than in the case $q=2\left(\right.$ take $\left.T^{\star}=T\right)$. 
When the feedback is locally distributed in the domain $(\omega=(1 / 2-\lambda, 1 / 2+\lambda)$ with $\lambda<1 / 2)$ and $T$ is not an exceptional value:

$$
\frac{1}{T} \notin \bigcup_{p=1}^{q-1} \frac{q}{p} \mathbb{N}
$$

then there exists $N_{T}$ such that for all $t \in[0,1], a(t)+a(t+1)+\cdots+a\left(t+N_{r}\right) \geq 1$. (compare with Lem. 7.1), and we deduce that (5.7) is satisfied with $T^{*}:=N_{r}+1$, (the proof is the same as in the case $q=2$ ).

When

$$
\frac{1}{T} \in \bigcup_{p=1}^{q-1} \frac{q}{p} \mathbb{N} \text { and }(q-1) T<2 \lambda,
$$

then first we apply Theorem 5.1 and we obtain that for all solutions $\phi$ of (2.6):

$$
E_{\phi}(0) \leq C \int_{\eta}^{T^{*}-\eta} \int_{\omega} \phi_{t}^{2}(x, t) \mathrm{d} x \mathrm{~d} t
$$

where $T^{*}$ is chosen like in the remark following Theorem 2.4. Then applying the method used for prove Theorem 5.2 and the fact that $(q-1) T<2 \lambda$, one obtain $(2.10)$.

At last, if

$$
\frac{1}{T} \in \bigcup_{p=1}^{q-1} \frac{q}{p} \mathbb{N} \text { and }(q-1) T>2 \lambda,
$$

then following Section 10.4 we can construct a solution of (1.2) whose energy remains constant with time.

\section{Proof of Proposition 10.1 (Link Between stabilization And observability) \\ 13.1. Equation (10.3) implies (10.4)}

Assume (10.3). Given $\left(u^{0}, u^{1}\right) \in H_{0}^{1}(\Omega) \times L^{2}(\Omega)$, we want to prove that the energy of the solution $u$ of $(10.1)$ decays exponentially to 0 . Set $k \in \mathbb{N}$ ( $k$ will be chosen later $)$ : since

$$
\int_{0}^{k T^{*}} \int_{\omega} a(t) u_{t}^{2} \mathrm{~d} x \mathrm{~d} t=E_{u}(0)-E_{u}\left(k T^{*}\right) \leq E_{u}(0),
$$

there exists some $p \in 0, \cdots, k-1$ such that

$$
\int_{p T^{*}}^{(p+1) T^{*}} \int_{\omega} a(t) u_{t}^{2} \mathrm{~d} x \mathrm{~d} t \leq \frac{1}{k} E_{u}(0) .
$$

Choose $\left(\phi_{0}, \phi_{1}\right)$ such that the solution of $(2.6)$ satisfies $\left(\phi\left(p T^{*}\right), \phi_{t}\left(p T^{*}\right)\right)=\left(u\left(p T^{*}\right), u_{t}\left(p T^{*}\right)\right)$, and consider $w:=u-\phi: w$ satisfies

$$
\left\{\begin{array}{l}
w_{t t}-\Delta w=-a(t) \chi_{\omega}(x) u_{t} \text { on } \Omega \times \mathbb{R}_{+} \\
w=0 \text { on } \partial \Omega \times \mathbb{R}_{+} \\
w\left(p T^{*}\right)=0=w_{t}\left(p T^{*}\right)
\end{array}\right.
$$

Note that

$$
\int_{\Omega} w_{t}^{2} \mathrm{~d} x \leq 2 \int_{\Omega} u_{t}^{2}+\phi_{t}^{2} \mathrm{~d} x \leq 8 E_{u}(0) .
$$


Hence

$$
\forall t \geq p T^{*}, E_{w}(t)-E_{w}\left(p T^{*}\right)=-\int_{p T^{*}}^{t} \int_{\omega} a(t) u_{t} w_{t} \mathrm{~d} x \mathrm{~d} t
$$

Thus for all $t \in\left(p T^{*},(p+1) T^{*}\right)$,

$$
\begin{aligned}
E_{w}(t) & \leq\left(\int_{p T^{*}}^{(p+1) T^{*}} \int_{\omega} a(t)^{2} u_{t}^{2} \mathrm{~d} x \mathrm{~d} t\right)^{1 / 2}\left(\int_{p T^{*}}^{(p+1) T^{*}} \int_{\omega} w_{t}^{2} \mathrm{~d} x \mathrm{~d} t\right)^{1 / 2} \\
& \leq \sup \sqrt{a}\left(\frac{1}{k} E_{u}(0)\right)^{1 / 2}\left(8 T^{*} E_{u}(0)\right)^{1 / 2}=\frac{C}{\sqrt{k}} E_{u}(0) .
\end{aligned}
$$

Hence using the assumption (10.3) on the problem (2.6), the periodicity of $a$, and (13.2), we obtain that

$$
\begin{aligned}
\forall t \geq p T^{*}, E_{\phi}(t)=E_{\phi}\left(p T^{*}\right) & \leq C \int_{p T^{*}}^{(p+1) T^{*}} \int_{\omega} a(t) \phi_{t}^{2} \mathrm{~d} x \mathrm{~d} t \\
& \leq C \int_{p T^{*}}^{(p+1) T^{*}} \int_{\omega}\left(2 a(t) y^{\prime 2}+2 a(t) w_{t}^{2}\right) \mathrm{d} x \mathrm{~d} t \\
& \leq \frac{2 C}{k} E_{u}(0)+2 C \sup a \frac{2 C T^{*}}{\sqrt{k}} E_{u}(0)
\end{aligned}
$$

Hence using (13.2) and (13.3), we obtain that

$$
\forall t \in\left(p T^{*},(p+1) T^{*}\right), E_{u}(t) \leq 2 E_{\phi}(t)+2 E_{w}(t) \leq\left(\frac{C_{1}}{k}+\frac{C_{2}}{\sqrt{k}}\right) E_{u}(0) \leq \frac{1}{2} E_{u}(0)
$$

if $k$ is large enough. Hence

$$
E_{u}\left(T_{1}=k T^{*}\right) \leq \frac{1}{2} E_{u}(0)
$$

and this implies (10.4) using the periodicity of $a$.

\subsection{Equation (10.4) implies (10.3)}

Set $\left(\phi_{0}, \phi_{1}\right) \in H_{0}^{1}(\Omega) \times L^{2}(\Omega)$. Let $y$ be the solution of $(10.1)$ with $\left(u^{0}, u^{1}\right)=\left(\phi_{0}, \phi_{1}\right)$. Set $T^{*}>0$. We deduce from (10.4) that

$$
\int_{0}^{T^{*}} \int_{\omega} a u_{t}^{2} \mathrm{~d} x \mathrm{~d} t=E_{u}(0)-E_{u}(t) \geq\left(1-\mathrm{e}^{\delta\left(T_{1}-T^{*}\right)}\right) E_{u}(0) \geq \frac{1}{2} E_{u}(0)
$$

if $T^{*}$ is large enough. Choose such $T^{*}$. (Note that $T^{*}$ is independent of $\left(\phi_{0}, \phi_{1}\right)$.) Then we want to prove that there exists $C>0$ (independent of $\left.\left(\phi_{0}, \phi_{1}\right)\right)$ such that

$$
E_{u}(0)=E_{\phi}(0) \leq C \int_{0}^{T^{*}} \int_{\omega} a \phi_{t}^{2} \mathrm{~d} x \mathrm{~d} t
$$

Using (13.4), it is sufficient to prove that there exists $C>0$ such that

$$
\int_{0}^{T^{*}} \int_{\omega} a u_{t}^{2} \mathrm{~d} x \mathrm{~d} t \leq C \int_{0}^{T^{*}} \int_{\omega} a \phi_{t}^{2} \mathrm{~d} x \mathrm{~d} t
$$


Once again consider $w=u-\phi ; w$ is solution of

$$
\left\{\begin{array}{l}
w_{t t}-\Delta w+a(t) \chi_{\omega}(x) w_{t}=-a(t) \chi_{\omega}(x) \phi_{t} \text { on } \Omega \times \mathbb{R}_{+}, \\
w=0 \text { on } \partial \Omega \times \mathbb{R}_{+} \\
w(0)=0=w_{t}(0)
\end{array}\right.
$$

Hence

$$
E_{w}(t)=E_{w}(0)-\int_{0}^{T^{*}} \int_{\omega} a w_{t}^{2} \mathrm{~d} x \mathrm{~d} t-\int_{0}^{T^{*}} \int_{\omega} a \phi_{t} w_{t} \mathrm{~d} x \mathrm{~d} t \leq \int_{0}^{T^{*}} \int_{\omega} a\left|\phi_{t} w_{t}\right| \mathrm{d} x \mathrm{~d} t
$$

Set $\varepsilon>0$ : then we have

$$
\begin{aligned}
\int_{\Omega} w_{t}^{2} \mathrm{~d} x \leq 2 E_{w}(t) & \leq 2 \int_{0}^{T^{*}} \int_{\omega} a\left|\phi_{t} w_{t}\right| \mathrm{d} x \mathrm{~d} t \\
& \leq \frac{1}{\varepsilon} \int_{0}^{T^{*}} \int_{\omega} a \phi_{t}^{2} \mathrm{~d} x \mathrm{~d} t+\varepsilon \int_{0}^{T^{*}} \int_{\omega} a w_{t}^{2} \mathrm{~d} x \mathrm{~d} t \\
& \leq \frac{1}{\varepsilon} \int_{0}^{T^{*}} \int_{\omega} a \phi_{t}^{2} \mathrm{~d} x \mathrm{~d} t+\varepsilon \sup a \int_{0}^{T^{*}} \int_{\Omega} w_{t}^{2} \mathrm{~d} x \mathrm{~d} t
\end{aligned}
$$

hence integrating over $\left(0, T^{*}\right)$ and choosing $\varepsilon>0$ small enough such that $\varepsilon T^{*} \sup a<1$, we find that there exists $C>0$ such that

$$
\int_{0}^{T^{*}} \int_{\Omega} w_{t}^{2} \mathrm{~d} x \mathrm{~d} t \leq C \int_{0}^{T^{*}} \int_{\omega} a \phi_{t}^{2} \mathrm{~d} x \mathrm{~d} t
$$

Note that $C$ depends on $T^{*}$ but not on $\phi$. Now we can easily conclude

$$
\int_{0}^{T^{*}} \int_{\omega} a u_{t}^{2} \mathrm{~d} x \mathrm{~d} t \leq 2 \int_{0}^{T^{*}} \int_{\omega}\left(a \phi_{t}^{2}+a w_{t}^{2}\right) \mathrm{d} x \mathrm{~d} t \leq C^{\prime} \int_{0}^{T^{*}} \int_{\omega} a \phi_{t}^{2} \mathrm{~d} x \mathrm{~d} t
$$

and that gives (10.3) through (13.4). The proof of Proposition 10.1 is complete.

The authors are grateful to V. Komornik and E. Zuazua for their comments and suggestions on this work.

\section{REFERENCES}

[1] Z. Artstein and E.F. Infante, On the asymptotic stability of oscillators with unbounded damping. Quart. Appl. Math. 34 (1976) 195-199.

[2] C. Bardos, G. Lebeau and J. Rauch, Sharp sufficient conditions for the observation, control and stabilization of waves from the boundary. SIAM J. Control Optim. 30 (1992) 1024-1065.

[3] C. Bardos, G. Lebeau and J. Rauch, Un exemple d'utilisation des notions de propagation pour le contrôle et la stabilisation de problèmes hyperboliques, Nonlinear hyperbolic equations in applied sciences. Rend. Sem. Mat. Univ. Politec. Torino 1988, Special Issue (1989) 11-31.

[4] A. Bayliss and E. Turkel, Radiation boundary conditions for wave-like equations. Comm. Pure Appl. Math. 33 (1980) 707-725.

[5] A. Benaddi and B. Rao, Energy decay rate of wave equations with indefinite damping. J. Differential Equations 161 (2000) 337-357.

[6] C. Castro and S.J. Cox, Achieving arbitrarily large decay in the damped wave equation. SIAM J. Control Optim. 39 (2001) 1748-1755.

[7] P. Freitas and E. Zuazua, Stability results for the wave equation with indefinite damping. J. Differential Equations 132 (1996) 338-352. 
[8] A. Haraux, Une remarque sur la stabilisation de certains systèmes du deuxième ordre en temps, Publications du Laboratoire d'Analyse numérique. Université Pierre et Marie Curie (1988).

[9] A. Haraux, A generalized internal control for the wave equation in a rectangle. J. Math. Anal. Appl. 153 (1990) $190-216$.

[10] W.A. Harris Jr., P. Pucci and J. Serrin, Asymptotic behavior of solutions of a nonstandard second order differential equation. Differential Integral Equations 6 (1993) 1201-1215.

[11] L. Hatvani, On the stability of the zero solution of second order nonlinear differential equations. Acta Sci. Math. 32 (1971) 1-9.

[12] L. Hatvani and V. Totik, Asymptotic stability of the equilibrium of the damped oscillator. Differential Integral Equation 6 (1993) 835-848.

[13] L. Hatvani, T. Krisztin, V. Totik and Vilmos, A necessary and sufficient condition for the asymptotic stability of the damped oscillator. J. Differential Equations 119 (1995) 209-223.

[14] S. Jaffard, M. Tucsnak and E. Zuazua, Singular internal stabilization of the wave equation. J. Differential Equations 145 (1998) 184-215.

[15] V. Komornik and E. Zuazua A direct method for boundary stabilization of the wave equation. J. Math. Pures Appl. 69 (1990) 33-54.

[16] V. Komornik, Exact Controllability and Stabilization. The Multiplier Method. John Wiley, Chicester and Masson, Paris (1994).

[17] J. Lagnese, Decay of solutions of wave equations in a bounded region with boundary dissipation. J. Differential Equations $\mathbf{5 0}$ (1983) 163-182.

[18] J. Lagnese, Note on boundary stabilization of wave equation. SIAM J. Control Optim. 26 (1988) 1250-1256.

[19] I. Lasiecka and R. Triggiani, Uniform exponential decay in a bounded region with $L_{2}\left(0, T ; L_{2}(\Sigma)\right)$-feedback control in the Dirichlet boundary condition. J. Differential Equations 66 (1987) 340-390.

[20] I. Lasiecka and R. Triggiani, Uniform stabilization of the wave equation with Dirichlet or Neumann feedback control without geometrical conditions. Appl. Math. Optim. 25 (1992) 189-224.

[21] J.-L. Lions, Contrôlabilité exacte de systèmes distribués. C. R. Acad. Sci. Paris 302 (1986) $471-475$.

[22] J.-L. Lions, Contrôlabilité exacte, stabilisation et perturbations de systèmes distribués. Masson, RMA 8 (1988).

[23] J.-L. Lions, Exact controllability, stabilization adn perturbations for distributd systems. SIAM Rev. 30 (1988) 1-68.

[24] S. Marakuni, Asymptotic behavior of solutions of one-dimensional damped wave equations. Comm. Appl. Nonlin. Anal. 1 (1999) 99-116.

[25] P. Martinez, Precise decay rate estimates for time-dependent dissipative systems. Israël J. Math. 119 (2000) $291-324$.

[26] P. Martinez and J. Vancostenoble, Exact controllability in "arbitrarily short time" of the semilinear wave equation. Discrete Contin. Dynam. Systems (to appear).

[27] M. Nakao, On the time decay of solutions of the wave equation with a local time-dependent nonlinear dissipation. Adv. Math. Sci. Appl. 7 (1997) 317-331.

[28] K. Petersen, Ergodic Theory. Cambridge University Press, Cambridge, Studies in Adv. Math. 2 (1983).

[29] P. Pucci and J. Serrin, Precise damping conditions for global asymptotic stability for nonlinear second order systems. Acta Math. 170 (1993) 275-307.

[30] P. Pucci and J. Serrin, Precise damping conditions for global asymptotic stability for nonlinear second order systems, II. J. Differential Equations 113 (1994) 505-534.

[31] P. Pucci and J. Serrin, Asymptotic stability for intermittently controlled nonlinear oscillators. SIAM J. Math. Anal. 25 (1994) $815-835$.

[32] P. Pucci and J. Serrin, Asymptotic stability for nonautonomous dissipative wave systems. Comm. Pure Appl. Math. XLIX (1996) 177-216.

[33] P. Pucci and J. Serrin, Local asymptotic stability for dissipative wave systems. Israël J. Math. 104 (1998) 29-50.

[34] R.A. Smith, Asymptotic stability of $x^{\prime \prime}+a(t) x^{\prime}+x=0$. Quart. J. Math. Oxford (2) 12 (1961) $123-126$.

[35] L.H. Thurston and J.W. Wong, On global asymptotic stability of certain second order differential equations with integrable forcing terms. SIAM J. Appl. Math. 24 (1973) 50-61.

[36] J. Vancostenoble and P. Martinez, Optimality of energy estimates for the wave equation with nonlinear boundary velocity feedbacks. SIAM J. Control Optim. 39 (2000) 776-797.

[37] E. Zuazua, An introduction to the exact controllability for distributed systems, Textos et Notas 44, CMAF. Universidades de Lisboa (1990). 